\title{
SOME REMARKS ON THE UNROLLED QUANTUM GROUP OF $\mathfrak{s l}(2)$
}

\author{
FRANCESCO COSTANTINO, NATHAN GEER, AND BERTRAND PATUREAU-MIRAND
}

\begin{abstract}
In this paper we consider the representation theory of a non-standard quantization of $\mathfrak{s l}(2)$. This paper contains several results which have applications in quantum topology, including the classification of projective indecomposable modules and a description of morphisms between them. In the process of proving these results the paper acts as a survey of the known representation theory associated to this non-standard quantization of $\mathfrak{s l}(2)$. The results of this paper are used extensively in 4 to study Topological Quantum Field Theory (TQFT) and have connections with Conformal Field Theory (CFT).
\end{abstract}

\section{INTRODUCTION}

There are many different flavors of quantum $\mathfrak{s l}(2)$ based on a common algebraic presentations. In particular, these presentations depend on two features: (1) if the quantum parameter $q$ is generic or a root of unity and (2) what part of the center is killed. The associated representation theory varies widely when these features are changed. Two examples, when $q$ is a root of unity, are the finite-dimensional Hopf algebra commonly known as the small quantum group and the non-restricted quantum group obtained by specializing the De Concini-Kac form (for definitions of these algebras see [7]). The representation theory of the small quantum group leads to a modular category (in particular a finite, semi-simple, ribbon category) which can be used to construct 3-manifold invariants. On the other hand, the representation theory of the non-restricted quantum group contains an infinite class of modules called the cyclic modules.

In this paper we consider an intermediate quotient $\bar{U}_{q}^{H} \mathfrak{s l}(2)$, which we call the unrolled quantum group, leading to a category $\bar{U}_{q}^{H} \mathfrak{s l}(2)$-mod which is ribbon but not semi-simple or finite. This category has been used to construct quantum link and 3-manifold invariants in several papers $2,24,25,22,10,8,4$. These 3 -manifold invariants have powerful new properties, including asymptotic behavior related to the Volume Conjecture and novel quantum representation of mapping class groups (see [8, 4]). The existence of these properties is directly related to the unique representation theory discussed in this paper.

The Hopf algebra $\bar{U}_{q}^{H} \mathfrak{s l}(2)$ of this paper has an additional generator $H$ which is not in the usual quantum algebra associated to $\mathfrak{s l}(2)$. The element $H$ should be thought of as a logarithm of the usual generator $K$. The generator $H$ is used to define a braiding and a twist on a category of $\bar{U}_{q}^{H} \mathfrak{s l}(2)$-modules. In this category, $H$ is also responsible for the apparition of an infinite cyclic group of one dimensional invertible objects which play a key role in the topological applications.

The purpose of this paper is to give a survey of the known results about $\bar{U}_{q}^{H} \mathfrak{s l}(2)$-mod while proving some new useful results which have topological applications. In particular,

The first author's research was supported by French ANR project ANR-08-JCJC-0114-01. Research of the second author was partially supported by NSF grants DMS-1007197 and DMS-1308196. All the authors would like to thank the Erwin Schrödinger Institute for Mathematical Physics in Vienna for support during a stay in the Spring of 2014, where part of this work was done. 
we classify all indecomposable projective modules and define a modified trace on these objects (see Section 6). We give a "graded" quiver which describes the maps between the indecomposable projective modules (see Section 7). We also study the decomposition of the tensor product of certain indecomposable modules (see Section 8). These results are used in [4] in an essential way to build a TQFT for 3-manifolds equipped with a cohomology class. The category $\bar{U}_{q}^{H} \mathfrak{s l}(2)$-mod contains some indecomposable non-projective modules that are not studied in this paper (see for example their use in [9]). Instead here, we focus on semi-simple and projective modules that form together a sub tensor category (see Proposition 8.4.

The category of $\bar{U}_{q}^{H} \mathfrak{s l}(2)$-modules has a grading in the abelian group $\mathbb{C} / 2 \mathbb{Z}$ and its non semi-simple part is concentrated in degree $\overline{0}, \overline{1}$ blocks. These two blocks form a category similar to the category $\widetilde{U}_{q} \mathfrak{s l}(2)$-mod of representations of the standard small quantum group $\widetilde{U}_{q} \mathfrak{s l}(2)$. The category $\widetilde{U}_{q} \mathfrak{s l}(2)$-mod, equivalent to that of modules over the triplet vertex operator algebra $\mathcal{W}(p)$ (see [26, 28]), has been intensively studied in logarithmic conformal field theories (CFT) associated to the $(1, p)$ triplet algebras (see [18, 17, 6, 5, 12]). In particular, some results of Section 6 are similar to the analysis of projective modules in 18 .

The category $\bar{U}_{q}^{H} \mathfrak{s l}(2)$-mod has additional modules which do not appear in the representation theory of the small quantum group (in particular, the one dimensional invertible objects mentioned above). Moreover, conjecturally $\bar{U}_{q}^{H} \mathfrak{s l}(2)$-mod is equivalent to the category of representation of the vertex operator algebra called singlet vertex algebra $\mathcal{W}(2,2 p-1)$ (see 11, 11]). Understanding a deeper connection between the representation theory of this paper and CFT deserves some attention. For example, it would be interesting to compare the CFT representations of $S L(2, \mathbb{Z})$ (see [17) and more generally mapping class group representations (see [19]) with those obtained from $\bar{U}_{q}^{H} \mathfrak{s l}(2)$ in the TQFT of [4].

1.1. Acknowledgements. We would like to thank Simon Wood and Antun Milas for their useful comments on the relations with the theory of logarithmic CFTs and the organizers of the conference "Modern trends in topological quantum field theory" at the Erwin Schrödinger Institut (Vienna) for their kind invitation to the conference.

\section{A quANTIZATION OF $\mathfrak{s l}(2)$ AND ITS ASSOCIATED RIBBON CATEGORY}

In this section we recall the algebra $\bar{U}_{q}^{H} \mathfrak{s l}(2)$ and the category of modules over this algebra. Fix a positive integer $r$. Let $r^{\prime}=r$ if $r$ is odd and $r^{\prime}=\frac{r}{2}$ else. Let $\mathbb{C}$ be the complex numbers and $\ddot{\mathbb{C}}=(\mathbb{C} \backslash \mathbb{Z}) \cup r \mathbb{Z}$. Let $q=e^{\frac{\pi \sqrt{-1}}{r}}$ be a $2 r^{t h}$-root of unity. We use the notation $q^{x}=e^{\frac{\pi \sqrt{-1} x}{r}}$. For $n \in \mathbb{N}$, we also set

$$
\{x\}=q^{x}-q^{-x}, \quad[x]=\frac{\{x\}}{\{1\}}, \quad\{n\} !=\{n\}\{n-1\} \cdots\{1\} \quad \text { and } \quad[n] !=[n][n-1] \cdots[1]
$$

2.1. The Drinfel'd-Jimbo quantum group. Let $U_{q} \mathfrak{s l}(2)$ be the $\mathbb{C}$-algebra given by generators $E, F, K, K^{-1}$ and relations:

(1) $K K^{-1}=K^{-1} K=1, \quad K E K^{-1}=q^{2} E, \quad K F K^{-1}=q^{-2} F, \quad[E, F]=\frac{K-K^{-1}}{q-q^{-1}}$. 
The algebra $U_{q} \mathfrak{s l}(2)$ is a Hopf algebra where the coproduct, counit and antipode are defined by

$$
\begin{aligned}
& \Delta(E)=1 \otimes E+E \otimes K, \quad \varepsilon(E)=0, \quad S(E)=-E K^{-1}, \\
& \Delta(F)=K^{-1} \otimes F+F \otimes 1, \quad \varepsilon(F)=0, \quad S(F)=-K F, \\
& \Delta(K)=K \otimes K \quad \varepsilon(K)=1, \quad S(K)=K^{-1} .
\end{aligned}
$$

Let $\bar{U}_{q} \mathfrak{s l}(2)$ be the algebra $U_{q} \mathfrak{s l}(2)$ modulo the relations $E^{r}=F^{r}=0$. Also, let $\widetilde{U}_{q} \mathfrak{s l}(2)$ be the algebra $\bar{U}_{q} \mathfrak{s l}(2)$ modulo the relations $K^{2 r}=1$. These relations generate Hopf ideals so $\bar{U}_{q} \mathfrak{s l}(2)$ and $\widetilde{U}_{q} \mathfrak{s l}(2)$ inherit a Hopf algebra structure.

As we will now explain, the categories of modules over $U_{q} \mathfrak{s l}(2), \bar{U}_{q} \mathfrak{s l}(2)$ and $\widetilde{U}_{q} \mathfrak{s l}(2)$ have very different properties. Let $X$-mod be the tensor category of finite dimensional $X$-modules for $X$ equal to $U_{q} \mathfrak{s l}(2), \bar{U}_{q} \mathfrak{s l}(2)$ or $\widetilde{U}_{q} \mathfrak{s l}(2)$. The algebra $\widetilde{U}_{q} \mathfrak{s l}(2)$ is known as the small quantum group and has been well studied, see [7] and the references within. The algebra $U_{q} \mathfrak{s l}(2)$ is known as the De Concini-Kac quantum group. It and the category $U_{q} \mathfrak{s l}(2)$-mod have rich structures and have been studied in [13, 14, 15, 16. This category is not braided nor semi-simple and has an infinite number of simple modules called cyclic modules which are not highest weight modules. Finally, the category $\bar{U}_{q} \mathfrak{s l}(2)$-mod is not semi-simple nor braided and has an infinite number of non-isomorphic simple modules. However, one can easily modify $\bar{U}_{q} \mathfrak{s l}(2)$ and obtain a braided category of highest weight modules which has been used to construct invariants of links ([24]), of 3-manifolds ([8]) and TQFTs $(4)$. The aim of this paper is to give an overview of the algebraic results related to this modified quantization and prove a few straightforward results.

2.2. A modified version of $U_{q} \mathfrak{s l}(2)$. Let $U_{q}^{H} \mathfrak{s l}(2)$ be the $\mathbb{C}$-algebra given by generators $E, F, K, K^{-1}, H$ and relations in Equation (1) plus the relations:

$$
H K=K H, \quad[H, E]=2 E, \quad[H, F]=-2 F .
$$

The algebra $U_{q}^{H} \mathfrak{s l}(2)$ is a Hopf algebra where the coproduct, counit and antipode are defined by Equations (2) 4 and by

$$
\Delta(H)=H \otimes 1+1 \otimes H, \quad \varepsilon(H)=0, \quad S(H)=-H .
$$

Define $\bar{U}_{q}^{H} \mathfrak{s l}(2)$ to be the Hopf algebra $U_{q}^{H} \mathfrak{s l}(2)$ modulo the relations $E^{r}=F^{r}=0$.

Let $V$ be a finite dimensional $\bar{U}_{q}^{H} \mathfrak{s l}(2)$-module. An eigenvalue $\lambda \in \mathbb{C}$ of the operator $H: V \rightarrow V$ is called a weight of $V$ and the associated eigenspace is called a weight space. A vector $v$ in the $\lambda$-eigenspace of $H$ is a weight vector of weight $\lambda$, i.e. $H v=\lambda v$. We call $V$ a weight module if $V$ splits as a direct sum of weight spaces and $q^{H}=K$ as operators on $V$, i.e. $K v=q^{\lambda} v$ for any vector $v$ of weight $\lambda$. Let $\mathscr{C}$ be the category of finite dimensional weight $\bar{U}_{q}^{H} \mathfrak{s l}(2)$-modules.

Remark 2.1. The algebra $\bar{U}_{q}^{H} \mathfrak{s l}(2)$ does not have a requirement on $K^{r}$, allowing modules in $\mathscr{C}$ to have non-integral weights. The requirement $E^{r}=F^{r}=0$ forces modules to be highest weight modules. As we will see the generator $H$ is used to define a braiding on $\mathscr{C}$. Here the main point is that one must know the action of $H$ and not just the action of $K$ which acts as a kind of exponential of $H$.

Since $\bar{U}_{q}^{H} \mathfrak{s l}(2)$ is a Hopf algebra then $\mathscr{C}$ is tensor category where the unit $\mathbb{I}$ is the 1dimensional trivial module $\mathbb{C}$. Moreover, $\mathscr{C}$ is $\mathbb{C}$-linear: hom-sets are $\mathbb{C}$-modules, the composition and tensor product of morphisms are $\mathbb{C}$-bilinear, and $\operatorname{End}_{\mathscr{C}}(\mathbb{I})=\mathbb{C} \operatorname{Id}_{\mathbb{I}}$. When it is 
clear we denote the unit $\mathbb{I}$ by $\mathbb{C}$. We say a module $V$ is simple if has no proper submodules. If $V$ is simple then Schur's lemma implies that $\operatorname{End}_{\mathscr{C}}(V)=\mathbb{C} \operatorname{Id}_{V}$. If $\operatorname{End}_{\mathscr{C}}(V)=\mathbb{C I d}_{V}$ then for $f \in \operatorname{End}_{\mathscr{C}}(V)$ we denote $\langle f\rangle$ as the scalar determined by $f=\langle f\rangle \operatorname{Id}_{V}$.

We will now recall that the category $\mathscr{C}$ is a ribbon category. Let $V$ and $W$ be objects of $\mathscr{C}$. Let $\left\{v_{i}\right\}$ be a basis of $V$ and $\left\{v_{i}^{*}\right\}$ be a dual basis of $V^{*}=\operatorname{Hom}_{\mathbb{C}}(V, \mathbb{C})$. Then

$\overrightarrow{\operatorname{cov}}_{V}: \mathbb{C} \rightarrow V \otimes V^{*}$, given by $1 \mapsto \sum v_{i} \otimes v_{i}^{*}, \quad \overrightarrow{\mathrm{ev}}_{V}: V^{*} \otimes V \rightarrow \mathbb{C}$, given by $f \otimes w \mapsto f(w)$

are duality morphisms of $\mathscr{C}$. In 29] Ohtsuki truncates the usual formula of the $h$-adic quantum $\mathfrak{s l}(2) R$-matrix to define an operator on $V \otimes W$ by

$$
R=q^{H \otimes H / 2} \sum_{n=0}^{r-1} \frac{\{1\}^{2 n}}{\{n\} !} q^{n(n-1) / 2} E^{n} \otimes F^{n} .
$$

where $q^{H \otimes H / 2}$ is the operator given by

$$
q^{H \otimes H / 2}\left(v \otimes v^{\prime}\right)=q^{\lambda \lambda^{\prime} / 2} v \otimes v^{\prime}
$$

for weight vectors $v$ and $v^{\prime}$ of weights of $\lambda$ and $\lambda^{\prime}$. The $R$-matrix is not an element in $\bar{U}_{q}^{H} \mathfrak{s l}(2) \otimes \bar{U}_{q}^{H} \mathfrak{s l}(2)$, however the action of $R$ on the tensor product of two objects of $\mathscr{C}$ is a well defined linear map on such a tensor product. Moreover, $R$ gives rise to a braiding $c_{V, W}: V \otimes W \rightarrow W \otimes V$ on $\mathscr{C}$ defined by $v \otimes w \mapsto \tau(R(v \otimes w))$ where $\tau$ is the permutation $x \otimes y \mapsto y \otimes x$. Also, let $\theta$ be the operator given by

$$
\theta=K^{r-1} \sum_{n=0}^{r-1} \frac{\{1\}^{2 n}}{\{n\} !} q^{n(n-1) / 2} S\left(F^{n}\right) q^{-H^{2} / 2} E^{n}
$$

where $q^{-H / 2}$ is an operator defined by on a weight vector $v_{\lambda}$ by $q^{-H^{2} / 2} \cdot v_{\lambda}=q^{-\lambda^{2} / 2} v_{\lambda}$. Ohtsuki shows that the family of maps $\theta_{V}: V \rightarrow V$ in $\mathscr{C}$ defined by $v \mapsto \theta^{-1} v$ is a twist (see [27, 29]).

Now the ribbon structure on $\mathscr{C}$ yields right duality morphisms

$$
\overleftarrow{\mathrm{ev}}_{V}=\overrightarrow{\mathrm{ev}}_{V} c_{V, V^{*}}\left(\theta_{V} \otimes \operatorname{Id}_{V^{*}}\right) \text { and } \overleftarrow{\operatorname{coev}}_{V}=\left(\operatorname{Id}_{V^{*}} \otimes \theta_{V}\right) c_{V, V^{*}} \overrightarrow{\operatorname{cov}}_{V}
$$

which are compatible with the left duality morphisms $\left\{\overrightarrow{\operatorname{coev}}_{V}\right\}_{V}$ and $\left\{\overrightarrow{\mathrm{ev}}_{V}\right\}_{V}$. These duality morphisms are given by

$$
\begin{gathered}
\overleftarrow{\operatorname{coev}} V: \mathbb{C} \rightarrow V^{*} \otimes V, \text { where } 1 \mapsto \sum K^{r-1} v_{i} \otimes v_{i}^{*} \\
\overleftarrow{\mathrm{ev}}_{V}: V \otimes V^{*} \rightarrow \mathbb{C}, \text { where } v \otimes f \mapsto f\left(K^{1-r} v\right) .
\end{gathered}
$$

The quantum dimension $q \operatorname{dim}(V)$ of an object $V$ in $\mathscr{C}$ is the $q \operatorname{dim}(V)=\left\langle\overleftarrow{\mathrm{ev}}_{V} \circ \overrightarrow{\operatorname{cov}}_{V}\right\rangle=$ $\sum v_{i}^{*}\left(K^{1-r} v_{i}\right)$.

For $g \in \mathbb{C} / 2 \mathbb{Z}$, define $\mathscr{C}_{g}$ as the full sub-category of weight modules whose weights are

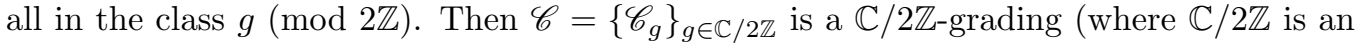
additive group): Let $V \in \mathscr{C}_{g}$ and $V^{\prime} \in \mathscr{C}_{g^{\prime}}$. Then the weights of $V \otimes V^{\prime}$ are congruent to $g+g^{\prime} \bmod 2 \mathbb{Z}$, and so the tensor product is in $\mathscr{C}_{g+g^{\prime}}$. Also if $g \neq g^{\prime}$ then $\operatorname{Hom}_{\mathscr{C}}\left(V, V^{\prime}\right)=0$ since morphisms in $\mathscr{C}$ preserve weights. Finally, for $f \in V^{*}=\operatorname{Hom}_{\mathbb{C}}(V, \mathbb{C})$ then by definition the action of $H$ on $f$ is given by $(H f)(v)=f(S(H) v)=-f(H v)$ and so $V^{*} \in \mathscr{C}_{-g}$. 


\section{Modified traces on the PROJeCtive MOdules.}

Let Proj be the full subcategory of $\mathscr{C}$ consisting of projective $\bar{U}_{q}^{H} \mathfrak{s l}(2)$-modules. The subcategory Proj is an ideal (see also [20]): it is closed under retracts (i.e. if $W \in$ Proj and $\alpha: X \rightarrow W$ and $\beta: W \rightarrow X$ satisfy $\beta \circ \alpha=\operatorname{Id}_{X}$, then $\left.X \in \operatorname{Proj}\right)$ and if $X$ is in $\mathscr{C}$ and $Y$ is in Proj then $X \otimes Y$ is in Proj.

For any objects $V, W$ of $\mathscr{C}$ and any endomorphism $f$ of $V \otimes W$, set

$$
\operatorname{ptr}_{L}(f)=\left(\overrightarrow{\mathrm{ev}}_{V} \otimes \operatorname{Id}_{W}\right) \circ\left(\operatorname{Id}_{V^{*}} \otimes f\right) \circ\left(\overleftarrow{\operatorname{coev}}_{V} \otimes \operatorname{Id}_{W}\right) \in \operatorname{End}_{\mathscr{C}}(W)
$$

and

$$
\operatorname{ptr}_{R}(f)=\left(\operatorname{Id}_{V} \otimes \overleftarrow{\mathrm{ev}}_{W}\right) \circ\left(f \otimes \operatorname{Id}_{W^{*}}\right) \circ\left(\operatorname{Id}_{V} \otimes \overrightarrow{\operatorname{coev}}_{W}\right) \in \operatorname{End}_{\mathscr{C}}(V)
$$

Definition 3.1. A trace on Proj is a family of linear functions

$$
\left\{\mathrm{t}_{V}: \operatorname{End}_{\mathscr{C}}(V) \rightarrow K\right\}
$$

where $V$ runs over all objects of Proj and such that the following two conditions hold.

(1) If $U \in \operatorname{Proj}$ and $W \in \mathrm{Ob}(\mathscr{C})$ then for any $f \in \operatorname{End}_{\mathscr{C}}(U \otimes W)$ we have

$$
\mathrm{t}_{U \otimes W}(f)=\mathrm{t}_{U}\left(\operatorname{ptr}_{R}(f)\right) \text {. }
$$

(2) If $U, V \in$ Proj then for any morphisms $f: V \rightarrow U$ and $g: U \rightarrow V$ in $\mathscr{C}$ we have

$$
\mathrm{t}_{V}(g \circ f)=\mathrm{t}_{U}(f \circ g) .
$$

\section{The Center of $\bar{U}_{q}^{H} \mathfrak{s l}(2)$}

The center of the small quantum group is known (see [17]) and its dimension is $3 r-1$. The following proposition is a description of a subalgebra of the center of $\bar{U}_{q}^{H} \mathfrak{s l}(2)$. Let $C$ be quantum Casimir element defined by

$$
C=F E+\frac{K q+K^{-1} q^{-1}}{\{1\}^{2}}=E F+\frac{K q^{-1}+K^{-1} q}{\{1\}^{2}} .
$$

Also, let $\mathcal{T}_{r}$ be the $r^{\text {th }}$ Chebyshev polynomial determined by $\mathcal{T}_{r}\left(\frac{X+X^{-1}}{2}\right)=\frac{X^{r}+X^{-r}}{2}$.

Proposition 4.1. The center of $\bar{U}_{q}^{H} \mathfrak{s l}(2)$ contains the $\mathbb{C}$-algebra generated by $C$ and $K^{ \pm r}$ with the relation $\mathcal{T}_{r}\left(\frac{\{1\}^{2}}{2} C\right)=-\frac{K^{r}+K^{-r}}{2}$.

Proof. First, it is easy to see that the elements $C$ and $K^{ \pm r}$ are central. Next, we will show the relation stated in the proposition holds. Using induction on $k \in \mathbb{N}$ one can show that

$$
\prod_{i=0}^{k-1}\left(C-\frac{q^{-2 i-1} K+q^{2 i+1} K^{-1}}{\{1\}^{2}}\right)=E^{k} F^{k}
$$

On the other hand, we have

$$
\begin{gathered}
2\left(\mathcal{T}_{r}\left(\frac{X+X^{-1}}{2}\right)-\mathcal{T}_{r}\left(\frac{Y+Y^{-1}}{2}\right)\right)=\left(X^{r}+X^{-r}\right)-\left(Y^{r}+Y^{-r}\right) \\
=X^{-r}\left(X^{r}-Y^{r}\right)\left(X^{r}-Y^{-r}\right)=\prod_{i=0}^{r-1} X^{-1}\left(X-q^{2 i} Y\right)\left(X-q^{-2 i} Y^{-1}\right) \\
=\prod_{i=0}^{r-1}\left(X+X^{-1}-Y q^{2 i}-Y^{-1} q^{-2 i}\right) .
\end{gathered}
$$


Combine the last expression with the fact that the product of Equation (13) vanishes for $k=r$ we obtain the following polynomial relation of degree $r$ for $C$ :

$$
2 \mathcal{T}_{r}\left(\frac{\{1\}^{2}}{2} C\right)-2 \mathcal{T}_{r}\left(\frac{q K+q^{-1} K^{-1}}{2}\right)=\prod_{i=0}^{r-1}\left(\{1\}^{2} C-\left(q^{2 i+1} K+q^{-2 i-1} K^{-1}\right)\right)=0 .
$$

Thus, $\mathcal{T}_{r}\left(\frac{\{1\}^{2}}{2} C\right)=-\frac{K^{r}+K^{-r}}{2}$.

It can be show that the center center of $\bar{U}_{q}^{H} \mathfrak{s l}(2)$ contains more complicated elements involving the element $H$. We don't need these elements in the rest of the paper.

\section{Simple $\bar{U}_{q}^{H} \mathfrak{s l}(2)$-MODULES}

For each $n \in\{0, \ldots, r-1\}$ let $S_{n}$ be the usual $(n+1)$-dimensional simple highest weight $\bar{U}_{q}^{H} \mathfrak{s l}(2)$-module with highest weight $n$. The module $S_{n}$ is a highest weight module with a highest weight vector $s_{0}$ such that $E s_{0}=0$ and $H s_{0}=n s_{0}$. Then $\left\{s_{0}, s_{1}, \ldots, s_{n}\right\}$ is a basis of $S_{n}$ where $F s_{i}=s_{i+1}, H . s_{i}=(n-2 i) s_{i}, E . s_{0}=0=F^{n+1} . s_{0}$ and $E . s_{i}=\frac{\{i\}\{n+1-i\}}{\{1\}^{2}} s_{i-1}$. The quantum dimension $S_{i}$ is $\operatorname{qdim}\left(S_{n}\right)=(-1)^{n} \frac{\{n+1\}}{\{1\}}$.

In $\widetilde{U}_{q} \mathfrak{s l}(2)$-mod the modules $S_{n}$ are the only simple modules up to isomorphism. However in $\mathscr{C}$, there is a $(n+1)$-dimensional simple $\bar{U}_{q}^{H} \mathfrak{s l}(2)$-module with highest weight $n+r$ which not isomorphic to $S_{i}$, as follows. For $k \in \mathbb{Z}$, let $\mathbb{C}_{k r}^{H}$ be the one dimensional modules where both $E$ and $F$ act by zero and $H$ acts by $k r$. The degree of $\mathbb{C}_{k r}^{H}$ is $k r \bmod 2$. Then $S_{n} \otimes \mathbb{C}_{k r}^{H}$ is the simple highest weight module with highest weight $n+k r$. As a $\widetilde{U}_{q} \mathfrak{s l}(2)$ module $\mathbb{C}_{k r}^{H}$ is isomorphic to the trivial module. The modules $\mathbb{C}_{k r}^{H}$ are important tools in the work of [8, 4]. We also use another notation to distinguish among these modules, those that are in the degree $\overline{0}$ part of $\mathscr{C}$ : we define for any $k \in \mathbb{Z}$,

$$
\sigma^{k}=\mathbb{C}_{2 k r^{\prime}}^{H} \in \mathscr{C}_{\overline{0}} \quad \text { where } \quad r^{\prime}=r / 2 \text { if } r \in 2 \mathbb{Z} \quad \text { and } \quad r^{\prime}=r \text { else. }
$$

Next we consider a larger class of finite dimensional highest weight modules: for each $\alpha \in \mathbb{C}$ we let $V_{\alpha}$ be the $r$-dimensional highest weight $\bar{U}_{q}^{H} \mathfrak{s l}(2)$-module of highest weight $\alpha+r-1$. The modules $V_{\alpha}$ has a basis $\left\{v_{0}, \ldots, v_{r-1}\right\}$ whose action is given by

$$
H . v_{i}=(\alpha+r-1-2 i) v_{i}, \quad E . v_{i}=\frac{\{i\}\{i-\alpha\}}{\{1\}^{2}} v_{i-1}, \quad F . v_{i}=v_{i+1} \text {. }
$$

For all $\alpha \in \mathbb{C}$, the quantum dimension of $V_{\alpha}$ is zero:

$$
\operatorname{qdim}\left(V_{\alpha}\right)=\sum_{i=0}^{r-1} v_{i}^{*}\left(K^{1-r} v_{i}\right)=\sum_{i=0}^{r-1} q^{(r-1)(\alpha+r-1-2 i)}=q^{(r-1)(\alpha+r-1)} \frac{1-q^{2 r}}{1-q^{2}}=0 .
$$

We say $V_{\alpha}$ is typical if $\alpha \in(\mathbb{C} \backslash \mathbb{Z}) \cup r \mathbb{Z}$, otherwise it is atypical. If $V_{\alpha}$ is typical then it is simple, since it is generated by any of the basis vectors $v_{i}$ (see Equation (15)).

Definition 5.1. The character of a weight module $V \in \mathscr{C}$ is $\chi(V)=\sum_{\alpha} \operatorname{dim}(V(\alpha)) X^{\alpha} \in$ $\mathbb{Z}[\mathbb{C}]$ where $V(\alpha)$ is the $\alpha$-eigenspace of the action of $H$ on $V$ and $X^{\alpha}$ is a notation for the element $\alpha \in \mathbb{C}$ seen in the group $\operatorname{ring} \mathbb{Z}[\mathbb{C}]$.

Let $[k]_{X}=X^{k-1}+X^{k-3}+\cdots+X^{-(k-1)}$. Then for $\alpha \in \ddot{\mathbb{C}}$ and $i \in\{0, \ldots, r-1\}$, one has

$$
\chi\left(V_{\alpha}\right)=X^{\alpha}[r]_{X} \quad \text { and } \quad \chi\left(S_{i}\right)=[i+1]_{X} .
$$


Let $V, W \in \mathscr{C}$ and define

(17) $\Phi_{V, W}=\left(\operatorname{Id}_{W} \otimes \overleftarrow{\mathrm{ev}}_{V}\right) \circ\left(c_{V, W} \otimes \operatorname{Id}_{V^{*}}\right) \circ\left(c_{W, V} \otimes \operatorname{Id}_{V^{*}}\right) \circ\left(\operatorname{Id}_{W} \otimes \overrightarrow{\operatorname{coev}}_{V}\right) \in \operatorname{End}(W)$

Theorem 5.2. (1) If $\bar{\alpha} \in \mathbb{C} / 2 \mathbb{Z} \backslash \mathbb{Z} / 2 \mathbb{Z}$ then $\mathscr{C}_{\bar{\alpha}}$ is semi-simple.

(2) If $\alpha, \beta \in \ddot{\mathbb{C}}=(\mathbb{C} \backslash \mathbb{Z}) \cup r \mathbb{Z}$ and $\alpha+\beta \notin \mathbb{Z}$ then $V_{\alpha} \otimes V_{\beta} \simeq \oplus_{k \in H_{r}} V_{\alpha+\beta+k}$ where $H_{r}=\{-(r-1),-(r-3), \ldots, r-1\}$.

(3) All the typical modules are projective.

Proof. Let $\alpha \in \mathbb{C} \backslash \mathbb{Z}$ and define $c_{\alpha}=\frac{q^{\alpha+r}+q^{-\alpha-r}}{\{1\}^{2}}$. Proposition 4.1 implies that $C$ satisfies the relation $\prod_{i=0}^{r-1}\left(C-c_{\alpha+2 i}\right)=0$ on $\mathscr{C}_{\bar{\alpha}}$. Since $c_{\alpha+2 i}-c_{\alpha+2 j}=\frac{\{i-j\}\{\alpha+r+i+j\}}{\{1\}^{2}}$, this polynomial has only simple roots. Hence any $W \in \mathscr{C}_{\bar{\alpha}}$ splits as the direct sum of the eigenspaces of $C$. It is enough to show that $W$ is semi-simple when $C$ acts by a scalar (say $c_{\alpha}$ ) on $W$. Let $V$ be a maximal semi-simple submodule of $W$ and suppose $V \neq$ $W$. The weights of $W$ differ by elements of $2 \mathbb{Z}$. In particular, they are totally ordered and there is a weight vector $w$ of $W \backslash V$ of maximal weight $\lambda$. Hence $E . w \in V$ and $F E . w=\left(C-\frac{K q+K^{-1} q^{-1}}{\{1\}^{2}}\right) \cdot w=0$ because it is proportional to $w$ and also in $V$. It follows that $\lambda=\alpha+r-1$ modulo 2. Then by Equation $(13), E^{r-1} F^{r-1} . w=\nu w$ where $\nu=\prod_{i=1}^{r-1}\left(c_{\alpha}-c_{\alpha-2 i}\right) \neq 0$. Thus, $E . w=\frac{1}{\nu} E^{r} F^{r-1} . w=0$ and $w$ is an highest weight vector. It follows that $w$ generates a module $V^{\prime}$ isomorphic to the simple module $V_{\lambda-r+1}$ where $V \cap V^{\prime}=\{0\}$. This contradicts the maximality of $V$ and so $V=W$.

The direct sum decomposition of $V_{\alpha} \otimes V_{\beta}$ follows from a straightforward calculation using the character formula for a typical module. Finally, we prove the last statement of the theorem in two cases: 1 ) if $V_{\alpha}$ is a typical module with $\alpha \in \mathbb{C} \backslash \mathbb{Z}$ then the previous parts of the theorem imply that $V_{\alpha}$ is projective. 2) If $V_{\alpha}$ is a typical module with $\alpha=r n$ then it can be shown (see Lemma 6.6 that the morphism $\Phi_{V_{\beta}, V_{r n}}$ defined in (17) is nonzero for any $\beta \in \mathbb{C} \backslash \mathbb{Z}$. This morphism can be decomposed into the composition $g \circ f$ where $f: V_{r n} \rightarrow V_{\beta} \otimes V_{r n} \otimes V_{\beta}^{*}$ and $g: V_{\beta} \otimes V_{r n} \otimes V_{\beta}^{*} \rightarrow V_{r n}$ are the obvious morphisms. But $V_{\beta} \otimes V_{r n} \otimes V_{\beta}^{*}$ is projective because it is of the form $V_{\beta} \otimes W$ with $V_{\beta}$ projective. Furthermore, $\frac{1}{\langle g \circ f\rangle}(g \circ f)=\operatorname{Id}_{V_{r n}}$. Since the class of projective modules is closed under retracts then $V_{r n}$ is projective.

Lemma 5.3. Every simple module of $\mathscr{C}$ is isomorphic to exactly one of the modules in the list:

- $S_{n} \otimes \mathbb{C}_{k r}^{H}$, for $n=0, \cdots, r-2$ and $k \in \mathbb{Z}$,

- $V_{\alpha}$ for $\alpha \in(\mathbb{C} \backslash \mathbb{Z}) \cup r \mathbb{Z}$.

Proof. Let $W$ be a simple $\bar{U}_{q}^{H} \mathfrak{s l}(2)$-module in $\mathscr{C}$. Then $W$ is uniquely determined, up to isomorphism, by its highest weight $\lambda \in \mathbb{C}$. The lemma follows from the fact that the highest weight of modules in the above list is in bijection with elements of $\mathbb{C}$.

Note in the above lemma the modules $\mathbb{C}_{k r}^{H}$ and $S_{r-1} \otimes \mathbb{C}_{k r}^{H}$ are obtained by the isomorphisms $\mathbb{C}_{k r}^{H} \cong S_{0} \otimes \mathbb{C}_{k r}^{H}$ and $S_{r-1} \otimes \mathbb{C}_{k r}^{H} \cong V_{k r}$, respectively.

Theorem 5.4. There exists a unique trace on Proj up to multiplication by an element of $\mathbb{C}$. In particular, there is a unique trace $\mathrm{t}=\left\{\mathrm{t}_{V}\right\}_{V \in \text { Proj }}$ on Proj such that for any $f \in \operatorname{End}_{\mathscr{C}}\left(V_{0}\right)$ we have $\mathrm{t}_{V_{0}}(f)=(-1)^{r-1}\langle f\rangle$. 
Proof. The proof follows from results of [20, 24]. Here we explain this proof without recalling the definitions given in these papers: In [24] we show that if $\alpha \in \mathbb{C} \backslash \frac{1}{2} \mathbb{Z}$ then $V_{\alpha}$ is an ambidextrous object in $\mathscr{C}$. In [20] we show that an ambidextrous object $J$ leads to the existence of a unique (up to a constant) trace $\mathrm{t}$ on the ideal $\mathcal{I}_{J}$ generated by $J$. When $J$ is simple then the trace is uniquely determined by the assignment $\mathrm{t}_{J}(f)=c\langle f\rangle$, where $c$ is a constant. Since Proj generated by any $V_{\alpha}$ with $\alpha \in \mathbb{C} \backslash \frac{1}{2} \mathbb{Z}$ then there exists a trace with the above property. Finally, since Proj is generated by $V_{0}$ the theorem follows. 5.4

We define the modified quantum dimension function as

$$
\mathrm{d}: \mathrm{Ob}(\operatorname{Proj}) \rightarrow K \quad \text { by } \mathrm{d}(V)=\mathrm{t}_{V}\left(\mathrm{Id}_{V}\right) .
$$

We will prove in Lemma 6.8 that the modified quantum dimension function is given by

$$
\mathrm{d}\left(V_{\alpha}\right)=(-1)^{r-1} \prod_{j=1}^{r-1} \frac{\{j\}}{\{\alpha+r-j\}}=(-1)^{r-1} \frac{r\{\alpha\}}{\{r \alpha\}}=\frac{(-1)^{r-1} r}{q^{(1-r) \alpha}+\cdots+q^{(r-3) \alpha}+q^{(r-1) \alpha}}
$$

for $\alpha \in \ddot{\mathbb{C}}$.

\section{Projective modules}

Recall that an highest weight vector $v \in V$ is a weight vector such that $E v=0$. We call a weight vector $v$ dominant if $(F E)^{2} v=0$ (in particular, a highest weight vector is dominant). It is well known that a highest weight vector $v$ of a module $V$ generates a submodule with basis $\left\{F^{i} v\right\}$. The following proposition describes the submodule generated by a dominant weight vector.

Proposition 6.1. Let $v \in V$ be a dominant vector of weight $i \in\{0,1, \ldots, r-2\}$ and let $j=r-2-i$. Consider the following $2 r$ vectors of $V$ defined by

$$
\begin{array}{cccc}
\mathrm{w}_{i}^{H}=v, \quad \mathrm{w}_{r-j}^{R}=E \mathrm{w}_{i}^{H}, & \mathrm{w}_{i}^{S}=F \mathrm{w}_{r-j}^{R}, \quad \mathrm{w}_{j-r}^{L}=F^{i+1} \mathrm{w}_{i}^{H}, \\
\mathrm{w}_{i-2 k}^{H}=F^{k} \mathrm{w}_{i}^{H} & \text { and } & \mathrm{w}_{i-2 k}^{S}=F^{k} \mathrm{w}_{i}^{S} & \text { for } k=\{0 \cdots i\}, \\
\mathrm{w}_{r-j+2 k}^{R}=E^{k} \mathrm{w}_{r-j}^{R} & \text { and } & \mathrm{w}_{j-2 k-r}^{L}=F^{k} \mathrm{w}_{j-r}^{L} & \text { for } k=\{0 \cdots j\} .
\end{array}
$$

Then the vector space they generate is a submodule of $V$ and the following relations holds in $V$ (whenever the involved vectors are defined):

$$
\begin{array}{ccc}
H \mathrm{w}_{k}^{X}=k \mathrm{w}_{k}^{X}, & K \mathrm{w}_{k}^{X}=q^{k} \mathrm{w}_{k}^{X} \text { for } X \in\{L, R, H, S\}, \\
E \mathrm{w}_{k}^{R}=\mathrm{w}_{k+2}^{R}, & F \mathrm{w}_{k}^{X}=\mathrm{w}_{k-2}^{X} \text { for } X \in\{H, S, L\}, \\
F \mathrm{w}_{-i}^{H}=\mathrm{w}_{j-r}^{L}, \quad E \mathrm{w}_{j-r}^{L}=\mathrm{w}_{-i}^{S}, \quad E \mathrm{w}_{j+r}^{R}=E \mathrm{w}_{i}^{S}=F \mathrm{w}_{-i}^{S}=F \mathrm{w}_{-j-r}^{L}=0 \\
E \mathrm{w}_{i-2 k}^{H}=\gamma_{i, k} \mathrm{w}_{i-2 k+2}^{H}+\mathrm{w}_{i-2 k+2}^{S}, & E \mathrm{w}_{i-2 k}^{S}=\gamma_{i, k} \mathrm{w}_{i-2 k+2}^{S} \\
F \mathrm{w}_{r-j+2 k}^{R}=-\gamma_{j, k} \mathrm{w}_{r-j+2 k-2}^{R} & \text { and } \quad E \mathrm{w}_{j-2 k-r}^{L}=-\gamma_{j, k} \mathrm{w}_{j-2 k-r+2}^{L}
\end{array}
$$

where $\gamma_{n, k}=[k][n-k+1]=\gamma_{n, n-k+1}$.

$$
\begin{array}{|lllllll}
\mathrm{w}_{-j-r}^{L} & \cdots & \mathrm{w}_{j-r}^{L} \swarrow_{-i}^{\mathrm{w}_{-i}^{H}} & \cdots & \mathrm{w}_{i}^{H} \searrow_{\mathrm{w}_{-j+r}^{R}} & \cdots & \mathrm{w}_{j+r}^{R} \\
& & \mathrm{w}_{-i}^{S} & \cdots & \mathrm{w}_{i}^{S} \swarrow
\end{array}
$$
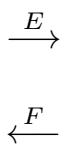

FIgURE 1 . The weight spaces structure of the module $P_{i}$ (here $j=r-2-i$ ). 
Proof. First, we show that the Relations $222-26)$ hold. The actions of $H$ and $K$ are easily deduced from their commutation relations with $E$ and $F$. The formulas in (23) are restatements of 20 - 21).

Let $c_{i}=\frac{q^{i+1}+q^{-i-1}}{\{1\}^{2}}=-\frac{q^{j+1}+q^{-j-1}}{\{1\}^{2}}$. We have $C \mathrm{w}_{i}^{H}=c_{i} \mathrm{w}_{i}^{H}+\mathrm{w}_{i}^{S}$. Since $F E \mathrm{w}_{i}^{S}=0$ then $C$ acts by the scalar $c_{i}$ on $\mathrm{w}_{i}^{S}$. As $C$ is central, we have

$$
E \mathrm{w}_{j-r}^{L}=E F \mathrm{w}_{-i}^{H}=\left(C-\frac{K q^{-1}+K^{-1} q}{\{1\}^{2}}\right) F^{i} \mathrm{w}_{i}^{H}=F^{i}\left(C-c_{i}\right) \mathrm{w}_{i}^{H}=F^{i} \mathrm{w}_{i}^{S}=\mathrm{w}_{-i}^{S} .
$$

Next, $F \mathrm{w}_{-j-r}^{L}=F\left(F^{j} F^{i+1} \mathrm{w}_{i}^{H}\right)=F^{r} \mathrm{w}_{i}^{H}=0$ so $C$ acts by $c_{i}$ on $\mathrm{w}_{-j-r}^{L}$. Then by induction on $k=0 \cdots j-1$,

$$
\begin{aligned}
E \mathrm{w}_{2 k-j-r}^{L} & =E F \mathrm{w}_{2 k-j-r+2}^{L}=\left(C-\frac{K q^{-1}+K^{-1} q}{\{1\}^{2}}\right) \mathrm{w}_{2 k-j-r+2}^{L} \\
& =\left(c_{i}-\frac{q^{2 k-j-r+1}+q^{-2 k+j+r-1}}{\{1\}^{2}}\right) \mathrm{w}_{2 k-j-r+2}^{L}=-[j-k][k+1] \mathrm{w}_{2 k-j-r+2}^{L} \\
& =-\gamma_{j, j-k} \mathrm{w}_{2 k-j-r+2}^{L} .
\end{aligned}
$$

By writing $2 k-j$ as $j-2(j-k)$ we obtain the second formula in $(26)$. Moreover, $C$ acts by $c_{i}$ on $\mathrm{w}_{2 k-j-r+2}^{L}=-\gamma_{j, j-k}^{-1} E \mathrm{w}_{2 k-j-r}^{L}$. Now $C$ also acts by $c_{i}$ on $\mathrm{w}_{i-2 k}^{S}=F^{k} \mathrm{w}_{i}^{S}$. This implies that for $k=1 \cdots i$,

$$
\begin{aligned}
E \mathrm{w}_{i-2 k}^{S} & =E F \mathrm{w}_{i-2 k+2}^{S}=\left(C-\frac{K q^{-1}+K^{-1} q}{\{1\}^{2}}\right) \mathrm{w}_{i-2 k+2}^{S} \\
& =\left(c_{i}-\frac{q^{i-2 k+1}+q^{-i+2 k-1}}{\{1\}^{2}}\right) \mathrm{w}_{i-2 k+2}^{S}=[k][i-k+1] \mathrm{w}_{i-2 k+2}^{S} .
\end{aligned}
$$

Since $E^{r}=0$ we have $E \mathrm{w}_{i}^{S}=E \frac{(-1)^{j}}{[i] !^{2}[j] !^{2}} E^{r-1} \mathrm{w}_{-r-j}^{L}=0$. This implies that $E F \mathrm{w}_{r-j}^{R}=$ $E \mathrm{w}_{i}^{S}=0$, so $C$ acts by the scalar $c_{i}$ on $\mathrm{w}_{r-j}^{R}$ and on $\mathrm{w}_{r-j+2 k}^{R}=E^{k} \mathrm{w}_{r-j}^{R}$. Using this, for $k=1 \cdots j$ we have

$$
\begin{aligned}
F \mathrm{w}_{r-j+2 k}^{R} & =F E \mathrm{w}_{r-j+2 k-2}^{R}=\left(C-\frac{K q+K^{-1} q^{-1}}{\{1\}^{2}}\right) \mathrm{w}_{r-j+2 k-2}^{R} \\
& =\left(c_{i}-\frac{q^{r-j+2 k-1}+q^{-r+j-2 k+1}}{\{1\}^{2}}\right) \mathrm{w}_{r-j+2 k-2}^{R}=-[k][j-k+1] \mathrm{w}_{r-j+2 k-2}^{R} .
\end{aligned}
$$

Since $F^{r}=0$ we have $F \mathrm{w}_{-i}^{S}=F \frac{(-1)^{j}}{([j] !)^{2}} F^{r-1} \mathrm{w}_{r+j}^{R}=0$. Then $C-c_{i}$ sends $\mathrm{w}_{i}^{H} \mapsto \mathrm{w}_{i}^{S}$ so it sends $\mathrm{w}_{i-2 k}^{H}=F^{k} \mathrm{w}_{i}^{H} \mapsto \mathrm{w}_{i-2 k}^{S}=F^{k} \mathrm{w}_{i}^{S}$. This implies that for $k=1 \cdots i$,

$$
\begin{aligned}
E \mathrm{w}_{i-2 k}^{H} & =E F \mathrm{w}_{i-2 k+2}^{H}=\left(C-\frac{K q^{-1}+K^{-1} q}{\{1\}^{2}}\right) \mathrm{w}_{i-2 k+2}^{H} \\
& =\left(c_{i}-\frac{q^{i-2 k+1}+q^{-i+2 k-1}}{\{1\}^{2}}\right) \mathrm{w}_{i-2 k+2}^{H}+\mathrm{w}_{i-2 k+2}^{S}=[k][i-k+1] \mathrm{w}_{i-2 k+2}^{H}+\mathrm{w}_{i-2 k+2}^{S} .
\end{aligned}
$$

Now $F \mathrm{w}_{-i}^{H}=F F^{i} \mathrm{w}_{i}^{H}=\mathrm{w}_{j-r}^{L}$. 
The final relation to check is $E \mathrm{w}_{j+r}^{R}=0$. Relation 25 implies

$$
E^{i} \mathrm{w}_{-i}^{H}=c \mathrm{w}_{i}^{H}+c^{\prime} \mathbf{w}_{i}^{S}
$$

where $c$ and $c^{\prime}$ are constants and $c \neq 0$. We have

$$
E^{r} \mathrm{w}_{-i}^{H}=E^{r-i}\left(c \mathrm{w}_{i}^{H}+c^{\prime} \mathbf{w}_{i}^{S}\right)=c E^{r-i-1} \mathbf{w}_{r-j}^{R}=c E \mathrm{w}_{r+j}^{R} .
$$

Thus, we have shown the Relations 19 -26 hold.

Finally the vector space generated by the vectors of this proposition is clearly stable by the generators of $\bar{U}_{q}^{H} \mathfrak{s l}(2)$ so it is a submodule of $V$.

Projective indecomposable weight modules in $\mathscr{C}_{\overline{0}} \cup \mathscr{C}_{\overline{1}}$ have a highest weight vector. The following proposition classifies the isomorphism classes of these modules.

Proposition 6.2. Let $i \in\{0,1, \ldots, r-2\}$ and let $j=r-2-i$. Denote the vectors of the canonical basis of $\mathbb{C}^{2 r}$ by

$\left(\mathrm{w}_{i}^{H}, \mathrm{w}_{i-2}^{H}, \ldots, \mathrm{w}_{-i}^{H}, \mathrm{w}_{r+j}^{R}, \mathrm{w}_{r+j-2}^{R}, \ldots, \mathrm{w}_{r-j}^{R}, \mathrm{w}_{j-r}^{L}, \mathrm{w}_{j-2-r}^{L}, \ldots, \mathrm{w}_{-j-r}^{L}, \mathrm{w}_{i}^{S}, \mathrm{w}_{i-2}^{S}, \ldots, \mathrm{w}_{-i}^{S}\right)$.

Then Formulas (19)-26) define a structure of weight module on $\mathbb{C}^{2 r}$ which we denote by $P_{i}$. Here $P_{r-1}=S_{r-1}=V_{0}$. The module $P_{i}$ is projective and indecomposable. Any projective indecomposable weight module $P \in \mathscr{C}_{\overline{0}} \cup \mathscr{C}_{\overline{1}}$ with highest weight $(k+1) r-i-2$ is isomorphic to $P_{i} \otimes \mathbb{C}_{k r}^{H}$.

Proof. A direct computation shows that the commutation relation $E F-F E=\frac{K-K^{-1}}{\{1\}}$ is satisfied on $P_{i}$, the other relations are consequences of the fact that $E$ and $F$ translate the weight spaces (see Figure 1). Hence Formulas $(19)-(26)$ define a structure of weight module on $P_{i}$.

Proposition 6.1 implies $P_{i}$ is a module which is generated by its dominant vector $\mathrm{w}_{i}^{H}$. Furthermore, if $V$ is any weight module then

$$
\operatorname{Hom}\left(P_{i}, V\right) \simeq\{v \in V: v \text { is dominant of weight } i\} .
$$

In particular, $\mathrm{w}_{i}^{H}$ and $\mathrm{w}_{i}^{S}$ are both dominant vector of $P_{i}$ of weight $i$, thus $\operatorname{End}\left(P_{i}\right)$ is a two dimensional vector space generated by Id : $\mathrm{w}_{i}^{H} \mapsto \mathrm{w}_{i}^{H}$ and the nilpotent map $x_{i}: \mathrm{w}_{i}^{H} \mapsto \mathrm{w}_{i}^{S}$ given by the action of $C-c_{i}$. This implies that $\operatorname{End}\left(P_{i}\right)$ is a local algebra and that $P_{i}$ is indecomposable (see [30, section 5.2]).

Let now $\phi: V \rightarrow P_{i}$ be a surjective map in $\mathscr{C}$. As $C$ is central, $V$ splits as the direct sum of the characteristic spaces of $C$ and only the summand $V_{i}$ of $V$ associated to the eigenvalue $c_{i}$ is not included in $\operatorname{ker} \phi$. We claim that $V_{i} \subset \operatorname{ker}\left(C-c_{i}\right)_{\mid V}^{2}$. Indeed Proposition 4.1 implies that on a module of $\mathscr{C}_{\overline{0}}, \mathcal{T}_{r}\left(\frac{\{1\}^{2}}{2} C\right)=-1=\mathcal{T}_{r}\left(\frac{q+q^{-1}}{2}\right)$ so $\prod_{i=0}^{r-1}\left(C-c_{2 i}\right)=0$ and on a module of $\mathscr{C}_{\overline{1}}$, we have $\mathcal{T}_{r}\left(\frac{\{1\}^{2}}{2} C\right)=1=\mathcal{T}_{r}\left(\frac{1+1}{2}\right)$ so $\prod_{i=0}^{r-1}\left(C-c_{2 i-1}\right)=0$. In both cases, all roots of the minimal polynomial of $C$ have multiplicity at most 2 and this proves that $V_{i} \subset \operatorname{ker}\left(C-c_{i}\right)_{\mid V}^{2}$. Any vector $v \in V_{i}$ of weight $i$ satisfy $\left(C-c_{i}\right)^{2} \cdot v=(F E)^{2} \cdot v=0$ thus is dominant. Let $v \in \phi^{-1}\left(\left\{\mathrm{w}_{i}^{H}\right\}\right)$. Then Proposition 6.1 implies that there is an unique $\psi \in \operatorname{Hom}\left(P_{i}, V_{i}\right)$ sending $\mathrm{w}_{i}^{H} \mapsto v$. Furthermore, $\phi \circ \psi\left(\mathrm{w}_{i}^{H}\right)=\mathrm{w}_{i}^{H}$ so $\phi \circ \psi=\operatorname{Id}_{P_{i}}$. Thus $\phi$ has a section and $P_{i}$ is projective.

For all $0 \leq i \leq r-1, \operatorname{Hom}\left(P_{i}, S_{i}\right) \simeq\left\{v \in S_{i}: v\right.$ is dominant of weight $\left.i\right\}$ is one dimensional generated by the surjective morphism $\pi_{i}$ sending $\mathrm{w}_{i}^{H}$ to a highest weight vector $v_{i}$ of $S_{i}$. Let $P^{\prime}$ be an indecomposable projective module and $\phi^{\prime}: P^{\prime} \rightarrow S$ a surjective map to a simple module (obtained by taking the quotient of $P^{\prime}$ by a maximal submodule). Then for some $k \in \mathbb{Z}$ and some $0 \leq i \leq r-1$, there exist an isomorphism 
$S \otimes \mathbb{C}_{r k}^{H} \simeq S_{i}$. If $i=r-1$ then $S$ is simple and projective. In this case $\phi^{\prime}$ has a section and is an isomorphism since $P^{\prime}$ is indecomposable. Assume now $i<r-1$ and let $P=P^{\prime} \otimes \mathbb{C}_{r k}^{H}$ which is also projective and indecomposable. Let $\phi=\phi^{\prime} \otimes \operatorname{Id}_{\mathbb{C}_{r k}^{H}}: P \rightarrow S_{i}$. Since $P$ is projective, there exists $\psi: P \rightarrow P_{i}$ such that $\phi=\pi_{i} \circ \psi$. Let $v \in P$ such that $\phi(v)=v_{i} \in S_{i}$. Then $\psi(v) \in \pi_{i}^{-1}\left(v_{i}\right) \cap P_{i}(i)=\mathrm{w}_{i}^{H}+\mathbb{C w}_{i}^{S}$ (here $P_{i}(i)$ is the weight space of weight $i$ of $P_{i}$ ). Hence $\psi(v)$ generates $P_{i}$ and $\psi$ is surjective. Finally, as $P_{i}$ is projective, $\psi$ has a section and is an isomorphism since $P$ is indecomposable. Thus we have $P^{\prime}=P \otimes \mathbb{C}_{-r k}^{H} \simeq P_{i} \otimes \mathbb{C}_{-r k}^{H}$.

The module $P_{i}$ is also an injective module in $\mathscr{C}$ which is self dual. Let $i \in\{0, \ldots, r-2\}$. After identifying both $S_{i}$ and $P_{i}$ with their duals, the map $\pi_{i}^{*}: S_{i} \rightarrow P_{i}$ is an injective morphism with image $\operatorname{Span}_{\mathbb{C}}\left(\mathrm{w}_{i}^{S}, \ldots, \mathrm{w}_{-i}^{S}\right)$. The quotient $\left(\operatorname{ker} \pi_{i}\right) / \pi_{i}^{*}\left(S_{i}\right)$ is isomorphic to $\left(\mathbb{C}_{r}^{H} \oplus \mathbb{C}_{-r}^{H}\right) \otimes S_{j}$ where $j=r-i-2$. As in the proof of Proposition 6.2, we let $x_{i}$ be the nilpotent endomorphism of $P_{i}$, that sends $\mathrm{w}_{i}^{H} \mapsto \mathrm{w}_{i}^{S}$. We have

$$
\operatorname{End}\left(P_{i}\right)=\mathbb{C} \operatorname{Id} \oplus \mathbb{C} x_{i}=\mathbb{C}\left[x_{i}\right] /\left(x_{i}^{2}\right) .
$$

Finally, the character of $P_{i}$ is given by

$$
\chi\left(P_{i}\right)=2[i+1]_{X}+\left(X^{r}+X^{-r}\right)[r-i-1]_{X}=[r]_{X}\left(X^{r-i-1}+X^{-r+i+1}\right) .
$$

Corollary 6.3. For all $\alpha \in \ddot{\mathbb{C}}, V_{\alpha} \otimes V_{-\alpha}$ is isomorphic to $V_{0} \otimes V_{0}$.

Proof. It follows from the previous proposition that the projective modules of $\mathscr{C}$ are determined up to isomorphism by their characters, so $V_{\alpha} \otimes V_{-\alpha}$ and $V_{0} \otimes V_{0}$ are isomorphic because they have the same character.

6.3

Recall the definitions of $\sigma^{k}$ and $r^{\prime}$ in Equation (14).

Corollary 6.4. Let $\alpha \in \mathbb{C} \backslash \mathbb{Z}$. Let $P \in \mathscr{C}_{\overline{0}}$ be a projective module, then there exist maps $f_{i}: P \rightarrow V_{0} \otimes \sigma^{n_{i}} \otimes V_{0}^{*}, g_{i}: V_{0} \otimes \sigma^{n_{i}} \otimes V_{0}^{*} \rightarrow P, f_{j}^{\prime}: P \rightarrow V_{\alpha+2 k_{j}} \otimes V_{-\alpha}$ and $g_{j}^{\prime}: V_{\alpha+2 k_{j}} \otimes V_{-\alpha} \rightarrow P$ such that

$$
\operatorname{Id}_{P}=\sum_{i} g_{i} f_{i}+\sum_{j} g_{j}^{\prime} f_{j}^{\prime}
$$

where $n_{i} \in \mathbb{Z}$ and $k_{j} \in \mathbb{Z} \backslash r^{\prime} \mathbb{Z}$.

Proof. Consider the epimorphism $f=\operatorname{Id}_{P} \otimes \overrightarrow{\mathrm{ev}}_{V_{-\alpha}}: P \otimes V_{-\alpha}^{*} \otimes V_{-\alpha} \rightarrow P$. Since $P$ is projective this morphism has a left inverse $g$, i.e. $f \circ g=\operatorname{Id}_{P}$. Now $P \otimes V_{-\alpha}^{*} \in \mathscr{C} \overline{\alpha+r-1}$ splits as a direct sum of modules isomorphic to $V_{\alpha+2 k}(k \in \mathbb{Z})$. This produces a factorization of $\operatorname{Id}_{P}$ through the modules $V_{\alpha+2 k} \otimes V_{-\alpha}$. Finally, if $k=r^{\prime} n \in r^{\prime} \mathbb{Z}$, then $V_{\alpha+2 k} \otimes V_{-\alpha} \simeq$ $\sigma^{n} \otimes V_{\alpha} \otimes V_{-\alpha} \simeq \sigma^{n} \otimes V_{0} \otimes V_{0} \simeq V_{0} \otimes \sigma^{n} \otimes V_{0}^{*}$.

The modified trace is non degenerate:

Proposition 6.5. Let $P \in \mathscr{C}$ be a projective module and $V \in \mathscr{C}$. Then the pairing

$$
\begin{array}{cl}
\operatorname{Hom}_{\mathscr{C}}(V, P) \times \operatorname{Hom}_{\mathscr{C}}(P, V) & \rightarrow \mathbb{C} \\
\left(h_{1}, h_{2}\right) & \mapsto \mathrm{t}_{P}\left(h_{1} h_{2}\right)
\end{array}
$$

is non degenerate.

Proof. Let $\alpha \in \mathbb{C} \backslash \mathbb{Z}$. Let $f: P \rightarrow V_{\alpha} \otimes W$ and $g: V_{\alpha} \otimes W \rightarrow P$ be morphisms of $\mathscr{C}$ such that $g f=\operatorname{Id}_{P}$. We show that for any non zero $h: P \rightarrow V$, there exists $h^{\prime}: V \rightarrow P$ such that $\mathrm{t}_{P}\left(h^{\prime} h\right) \neq 0$. Indeed, we have $h=h g f \neq 0$ thus we have a non trivial morphism 
$\left(h g \otimes \operatorname{Id}_{W^{*}}\right) \circ\left(\operatorname{Id}_{V_{\alpha}} \otimes \overrightarrow{\operatorname{coev}}_{W}\right): V_{\alpha} \rightarrow V \otimes W^{*}$. But $V_{\alpha}$ is in the category $\mathscr{C}_{\bar{\alpha}}$ which is semi-simple so the previous map has a left inverse $k: V \otimes W^{*} \rightarrow V_{\alpha}$. Then we have that

$$
\mathrm{t}_{V_{\alpha}}\left(k \circ\left(h g \otimes \operatorname{Id}_{W^{*}}\right) \circ\left(\operatorname{Id}_{V_{\alpha}} \otimes \overrightarrow{\operatorname{coe}}_{W}\right)\right)=\mathrm{d}\left(V_{\alpha}\right) \neq 0 .
$$

Let $k^{\prime}=\left(k \otimes \operatorname{Id}_{W}\right) \circ\left(\operatorname{Id}_{V} \otimes \overleftarrow{\operatorname{coev}}_{W}\right): V \rightarrow V_{\alpha} \otimes W$ and $h^{\prime}=g k^{\prime}$. Finally,

$\mathrm{t}_{P}\left(h^{\prime} h\right)=\mathrm{t}_{P}\left(g\left(k^{\prime} h\right)\right)=\mathrm{t}_{V_{\alpha} \otimes W}\left(\left(k^{\prime} h\right) g\right)=\mathrm{t}_{V_{\alpha}}\left(k \circ\left(h g \otimes \operatorname{Id}_{W^{*}}\right) \circ\left(\operatorname{Id}_{V_{\alpha}} \otimes \overrightarrow{\operatorname{coev}}_{W}\right)\right)=\mathrm{d}\left(V_{\alpha}\right) \neq 0$.

Lemma 6.6 (General Hopf links). Recall the map $\Phi$ given in Equation (17). For all $i, j \in\{0,1, \ldots, r-2\}$ and $\alpha, \beta \in \ddot{\mathbb{C}}=(\mathbb{C} \backslash \mathbb{Z}) \cup r \mathbb{Z}$, one has

$$
\begin{aligned}
\Phi_{V_{\beta}, V_{\alpha}}=\frac{(-1)^{r-1} r}{\mathrm{~d}\left(V_{\alpha}\right)} q^{\alpha \beta} \operatorname{Id}_{V_{\alpha}} & \Phi_{S_{i}, S_{j}}=(-1)^{i} \frac{\{(i+1)(j+1)\}}{\{j+1\}} \operatorname{Id}_{V_{j}} \\
\Phi_{S_{i}, V_{\alpha}}=\frac{\{(i+1) \alpha\}}{\{\alpha\}} \operatorname{Id}_{V_{\alpha}} & \Phi_{P_{i}, V_{\alpha}}=(-1)^{r-1} r \frac{q^{(r-1-i) \alpha}+q^{-(r-1-i) \alpha}}{\mathrm{d}\left(V_{\alpha}\right)} \operatorname{Id}_{V_{\alpha}}
\end{aligned}
$$

Moreover, recall the nilpotent $x_{j} \in \operatorname{End}\left(P_{j}\right)$ given by the action of $C-c_{j}$, then

$$
\Phi_{S_{i}, P_{j}}=(-1)^{i} \frac{\{(i+1)(j+1)\}}{\{j+1\}} \operatorname{Id}_{P_{j}}+(-1)^{i}\{1\}^{2} \frac{i\{(i+2)(j+1)\}-(i+2)\{i(j+1)\}}{\{j+1\}^{3}} x_{j},
$$

$$
\Phi_{V_{0}, P_{j}}=(-1)^{r+j} \frac{2 r\{1\}^{2}}{\{j+1\}^{2}} x_{j}, \quad \Phi_{P_{i}, P_{j}}=\frac{(-1)^{i} 2 r\{1\}^{2}}{\{j+1\}^{2}}\left(q^{(i+1)(j+1)}+q^{-(i+1)(j+1)}\right) x_{j} .
$$

Proof. For $\alpha \in \mathbb{C}$, let $\Psi_{\alpha}: \mathbb{Z}[\mathbb{C}] \rightarrow \mathbb{C}$ be the map sending $X^{z} \mapsto q^{\alpha z}$. We start by observing the following fact: Let $w$ be a highest weight vector of $W$ of weight $\alpha$, then

$$
\Phi_{V, W}(w)=\Psi_{\alpha+1-r}(\chi(V)) w .
$$

Indeed, the map $\Phi_{V, W}$ is given by the partial quantum trace of $c_{V, W} \circ c_{W, V}$. A standard argument shows that on a highest weight vector, this partial trace only depends of the Cartan part $q^{H \otimes H / 2}$ of the $R$-matrix. The identity then follows from a direct computation. A detailed presentation of an analogous computation is given in [21, Proposition 2.2]. Equation (32) implies that if $W$ is simple then $\Phi_{V, W}=\Psi_{\alpha+1-r}(\chi(V)) \operatorname{Id}_{W}$.

Equations (28) and (29) follow from Equation (32). For example, $\Phi_{P_{i}, V_{\alpha}}=\lambda \operatorname{Id}_{V_{\alpha}}$ where

$$
\lambda=\Psi_{\alpha}\left(\chi\left(P_{i}\right)\right)=\Psi_{\alpha}\left([r]_{X}\left(X^{r-i-1}+X^{-r+i+1}\right)\right)=\frac{(-1)^{r-1} r}{\mathrm{~d}\left(V_{\alpha}\right)}\left(q^{(r-1-i) \alpha}+q^{-(r-1-i) \alpha}\right) .
$$

Similarly, to compute $\Phi_{S_{1}, P_{j}}$ observe that any endomorphism of $P_{j}$ is of the form $a \operatorname{Id}+b x_{j} \in \operatorname{End}\left(P_{j}\right)=\mathbb{C}\left[x_{j}\right] /\left(x_{j}^{2}\right)$. Computing as above,

$$
\Phi_{S_{1}, P_{j}}\left(\mathrm{w}_{j}^{S}\right)=a \mathrm{w}_{j}^{S}
$$

where $\mathrm{w}_{j}^{S}$ is the highest weight vector of $P_{j}$ and $a=\Psi_{j+1-r}\left(X+X^{-1}\right)=-\left(q^{j+1}+q^{-j-1}\right)$. We now compute $b$. Recall that $S_{1}$ is generated by two weight vectors $\mathrm{s}_{0}, \mathrm{~s}_{1}$ and $E . \mathrm{s}_{1}=\mathrm{s}_{0}$, $F . \mathrm{s}_{0}=\mathrm{s}_{1}, H . \mathrm{s}_{i}=(-1)^{i} \mathrm{~s}_{i}$. In general,

$$
c_{W, V}=\tau \circ R=\tau \circ q^{H \otimes H / 2}\left(\operatorname{Id} \otimes \operatorname{Id}+\left(q-q^{-1}\right) E \otimes F+\cdots\right) .
$$


So

$$
\begin{gathered}
c_{S_{1}, P_{j}} \circ c_{P_{j}, S_{1}}\left(\mathbf{w}_{j}^{H} \otimes \mathbf{s}_{0}\right)=c_{S_{1}, P_{j}}\left(q^{\frac{j}{2}} \mathbf{s}_{0} \otimes \mathbf{w}_{j}^{H}+q^{-\frac{j+2}{2}}\left(q-q^{-1}\right) \mathbf{s}_{1} \otimes \mathbf{w}_{j+2}^{R}\right) \\
=\left(q^{j} \mathbf{w}_{j}^{H}+\left(q-q^{-1}\right)^{2} q^{-1} \mathbf{w}_{j}^{S}\right) \otimes \mathbf{s}_{0}+\cdots \otimes \mathbf{s}_{1} \\
c_{S_{1}, P_{j}} \circ c_{P_{j}, S_{1}}\left(\mathbf{w}_{j}^{H} \otimes \mathbf{s}_{1}\right)=c_{S_{1}, P_{j}}\left(q^{-\frac{j}{2}} \mathbf{s}_{1} \otimes \mathbf{w}_{j}^{H}\right)=q^{-j} \mathbf{w}_{j}^{H} \otimes \mathbf{s}_{1}+\cdots \otimes \mathbf{s}_{0} .
\end{gathered}
$$

When taking the quantum trace with respect to $S_{1}$ (i.e. the trace on $S_{1}$ of the endomorphism composed with Id $\otimes K^{1-r}$, we then get that

$\Phi_{S_{1}, P_{j}}\left(\mathrm{w}_{j}^{H}\right)=-q\left(q^{j} \mathbf{w}_{j}^{H}+\left(q-q^{-1}\right)^{2} q^{-1} \mathbf{w}_{j}^{S}\right)-q^{-1} \cdot q^{-j} \mathbf{w}_{j}^{H}=-\left(q^{j+1}+q^{-j-1}\right) \mathbf{w}_{j}^{H}-\left(q-q^{-1}\right)^{2} \mathbf{w}_{j}^{S}$ and we get $a=-\left(q^{j+1}+q^{-j-1}\right), b=-\left(q-q^{-1}\right)^{2}$. We have

$$
\Phi_{S_{1}, P_{j}} \circ \Phi_{S_{i}, P_{j}}=\Phi_{S_{1} \otimes S_{i}, P_{j}}=\Phi_{S_{i+1}, P_{j}}+\Phi_{S_{i-1}, P_{j}}
$$

so $\Phi_{S_{i}, P_{j}}$ is determined by the recurrence relations

$$
\Phi_{S_{i+1}, P_{j}}=\left(a+b x_{j}\right) \Phi_{S_{i}, P_{j}}-\Phi_{S_{i-1}, P_{j}}, \quad \Phi_{S_{1}, P_{j}}=a+b x_{j} \quad \text { and } \quad \Phi_{S_{0}, P_{j}}=1 .
$$

Solving for $\Phi_{S_{i}, P_{j}}$ we have the unique solution

$\Phi_{S_{i}, P_{j}}=\frac{(-1)^{i}}{\{j+1\}}\left(\{(i+1)(j+1)\} \operatorname{Id}_{P_{j}}+\frac{\{1\}^{2} x_{j}}{\{j+1\}^{2}}(i\{(i+2)(j+1)\}-(i+2)\{i(j+1)\})\right)$.

In particular, for $i=r-1, S_{i}=V_{0}$ and we get

$$
\Phi_{V_{0}, P_{j}}=(-1)^{r+j} \frac{2 r\{1\}^{2}}{\{j+1\}^{2}} x_{j} .
$$

Finally the character formulas give the isomorphism of projective modules:

$$
V_{0} \otimes S_{r-i-1}=V_{0} \otimes S_{r-i-3} \oplus P_{i} .
$$

Thus,

$$
\Phi_{P_{i}, P_{j}}=\frac{(-1)^{i} 2 r\{1\}^{2}}{\{j+1\}^{2}}\left(q^{(i+1)(j+1)}+q^{-(i+1)(j+1)}\right) x_{j} .
$$

Lemma 6.7. If $P$ is a projective module then $\mathrm{t}_{P}\left(\Phi_{V_{0}, P}\right)=\mathrm{t}_{V_{0}}\left(\Phi_{P, V_{0}}\right)=(-1)^{r-1}\left\langle\Phi_{P, V_{0}}\right\rangle$.

Proof. From the properties of a trace in Definition 3.1 we have

$$
\begin{gathered}
\mathrm{t}_{P}\left(\Phi_{V_{0}, P}\right)=\mathrm{t}_{P}\left(\operatorname{ptr}_{R}\left(c_{P, V_{0}} c_{V_{0}, P}\right)\right)=\mathrm{t}_{P \otimes V_{0}}\left(c_{V_{0}, P} c_{P, V_{0}}\right)=\mathrm{t}_{V_{0} \otimes P}\left(c_{P, V_{0}} c_{V_{0}, P}\right) \\
=\mathrm{t}_{V_{0}}\left(\operatorname{ptr}_{R}\left(c_{P, V_{0}} c_{V_{0}, P}\right)\right)=\mathrm{t}_{V_{0}}\left(\Phi_{P, V_{0}}\right)=(-1)^{r-1}\left\langle\Phi_{P, V_{0}}\right\rangle
\end{gathered}
$$

where the last equality follows from Theorem 5.4

Lemma 6.8 (The modified trace on typical modules). Let $V_{\alpha}$ be a typical module. Then for any $f \in \operatorname{End}_{\mathscr{C}}\left(V_{\alpha}\right), \operatorname{tr}_{V_{\alpha}}(f)=\mathrm{d}\left(V_{\alpha}\right)\langle f\rangle$ where $\mathrm{d}\left(V_{\alpha}\right)$ is given in Equation (18).

Proof. First, since $V_{\alpha}$ is simple we have $\mathrm{t}_{V_{\alpha}}(f)=\langle f\rangle \mathrm{t}_{V_{\alpha}}\left(\operatorname{Id}_{V_{\alpha}}\right)=\mathrm{d}\left(V_{\alpha}\right)\langle f\rangle$ where $\mathrm{d}\left(V_{\alpha}\right)=$ $\mathrm{t}_{V_{\alpha}}\left(\operatorname{Id}_{V_{\alpha}}\right)$. From Lemma 6.7 we have $\mathrm{t}_{V_{\alpha}}\left(\Phi_{V_{0}, V_{\alpha}}\right)=\mathrm{t}_{V_{0}}\left(\Phi_{V_{\alpha}, V_{0}}\right)$.

So $\mathrm{d}\left(V_{\alpha}\right)\left\langle\Phi_{V_{0}, V_{\alpha}}\right\rangle=\mathrm{d}\left(V_{0}\right)\left\langle\Phi_{V_{\alpha}, V_{0}}\right\rangle$ where $\mathrm{d}\left(V_{0}\right)=(-1)^{r-1}$, and

$$
\mathrm{d}\left(V_{\alpha}\right)=\mathrm{d}\left(V_{0}\right) \frac{\left\langle\Phi_{V_{\alpha}, V_{0}}\right\rangle}{\left\langle\Phi_{V_{0}, V_{\alpha}}\right\rangle} .
$$

Finally, the formula for $\mathrm{d}\left(V_{\alpha}\right)$ follows from Lemma 6.6 
Lemma 6.9 (The modified trace on $P_{j}$ ). We have

$$
\mathrm{d}\left(P_{j}\right)=\mathrm{t}_{P_{j}}\left(\operatorname{Id}_{P_{j}}\right)=(-1)^{j+1}\left(q^{j+1}+q^{-j-1}\right) \quad \text { and } \quad \mathrm{t}_{P_{j}}\left(x_{j}\right)=(-1)^{j+1} \frac{\{j+1\}^{2}}{\{1\}^{2}} .
$$

Proof. As $V_{0}$ is projective, so are $V_{0} \otimes S_{r-j-1}$ and $V_{0} \otimes S_{r-j-3}$. Now by Proposition 6.2 indecomposable projective modules are determined by their highest weight so the isomorphism class of a projective module is determined by its character. Hence the character formulas imply that there exists an isomorphism of projective modules $V_{0} \otimes S_{r-j-1} \simeq V_{0} \otimes S_{r-j-3} \oplus P_{j}$. Taking the modified traces of the identities of these modules gives $\mathrm{d}\left(V_{0}\right) \mathrm{qdim}\left(S_{r-j-1}\right)=\mathrm{d}\left(V_{0}\right) \mathrm{qdim}\left(S_{r-j-3}\right)+\mathrm{d}\left(P_{j}\right)$. Since $q \operatorname{dim}\left(S_{i}\right)=(-1)^{i} \frac{\{i+1\}}{\{1\}}$ we have

$$
\begin{aligned}
\mathrm{d}\left(P_{j}\right) & =\mathrm{d}\left(V_{0}\right)\left(\operatorname{qdim}\left(S_{r-j-1}\right)-\operatorname{qdim}\left(S_{r-j-3}\right)\right)=(-1)^{j}([r-j]-[r-j-2]) \\
& =(-1)^{j+1}\left(q^{j+1}+q^{-j-1}\right) .
\end{aligned}
$$

Lemma 6.7 implies $\mathrm{t}_{P_{j}}\left(\Phi_{V_{0}, P_{j}}\right)=\mathrm{t}_{V_{0}}\left(\Phi_{P_{j}, V_{0}}\right)$. Then Lemma6.6 implies

$$
(-1)^{r+j} 2 r \mathrm{t}_{P_{j}}\left(x_{j}\right) \frac{\{1\}^{2}}{\{j+1\}^{2}}=(-1)^{r-1} r \frac{2}{\mathrm{~d}\left(V_{0}\right)} \mathrm{t}_{V_{0}}\left(\operatorname{Id}_{V_{0}}\right)
$$

which implies the second relation of the lemma.

Lemma 6.10 (Twist on $P_{j}$ ). The action of the twist on $P_{j}$ is given by

$$
\theta_{P_{j}}=(-1)^{j} q^{\frac{j^{2}+2 j}{2}}\left(1-(r-j-1) \frac{\{1\}^{2}}{\{j+1\}} x_{j}\right) .
$$

In particular, $\theta_{P_{j}}$ has infinite order.

Proof. The twist commutes with the map $\pi_{j}: P_{j} \rightarrow S_{j}$ and $\pi_{j} x_{j}=0$. Thus the twist on $P_{j}$ is given by $\theta_{P_{j}}=\theta_{S_{j}}\left(1+\lambda x_{j}\right)$ where $\theta_{S_{j}}=(-1)^{j} q^{\frac{j^{2}+2 j}{2}}$ is the scalar action of the twist on $S_{j}$. Hence

$$
\mathrm{t}\left(\theta_{P_{j}}\right)=-q^{\frac{j^{2}+2 j}{2}}\left(q^{j+1}+q^{-j-1}+\lambda \frac{\{j+1\}^{2}}{\{1\}^{2}}\right) .
$$

Finally we use again the module $V_{0} \otimes S_{r-j-1} \simeq V_{0} \otimes S_{r-j-3} \oplus P_{j}$ to color the unknot with framing +1 . Its double is the Hopf link with both components having framing +1 and this gives

$$
\theta_{V_{0}} \theta_{S_{r-j-1}} . \mathrm{t}\left(\Phi\left(S_{r-j-1}, V_{0}\right)\right)=\theta_{V_{0}} \theta_{S_{r-j-3}} . \mathrm{t}\left(\Phi\left(S_{r-j-3}, V_{0}\right)\right)+\mathrm{t}\left(\theta_{P_{j}}\right)
$$

Hence

$$
\begin{aligned}
\mathrm{t}\left(\theta_{P_{j}}\right) & =\theta_{V_{0}} \mathrm{~d}\left(V_{0}\right)\left((r-j) \theta_{S_{r-j-1}}-(r-j-2) \theta_{S_{r-j-3}}\right) \\
& =-q^{j^{2} / 2-1}\left((r-j)-(r-j-2) q^{-2 j-2}\right) \\
& =-q^{\frac{j^{2}+2 j}{2}}\left(-(r-j-2) q^{j+1}+(r-j) q^{-j-1}\right)
\end{aligned}
$$

and this gives the announced formula for $\lambda$.

\section{The Algebra of PRoJective Modules}

In this section, we define and study two algebras encoding the maps between projective modules of $\mathscr{C}_{\overline{0}}$ and $\mathscr{C}_{\overline{1}}$ respectively. These are the algebras one would associate to curves in a $1+1+1$-TQFT which would be an extension of the $2+1$ TQFT given in [4]. 
7.1. Maps between indecomposable projective modules. We first describe the maps between indecomposable projective modules in the categories $\mathscr{C}_{\overline{0}}$ and $\mathscr{C}_{\overline{1}}$ :

Proposition 7.1. Let $i, \ell \in\{0, \ldots, r-2\}$ and $k \in \mathbb{Z}$. Let $P=\mathbb{C}_{k r}^{H} \otimes P_{\ell}$ be an indecomposable module, then any non zero map $P_{i} \rightarrow P$ is equal to $\lambda I_{i}+\mu x_{i}, \lambda \alpha_{i}^{+}$or $\lambda \alpha_{i}^{-}$where $\lambda, \mu \in \mathbb{C}$ and the maps $I_{i}, x_{i}, \alpha_{i}^{+}$and $\alpha_{i}^{-}$are uniquely determined by

$$
\begin{aligned}
I_{i}: P_{i} & \rightarrow P_{i} & x_{i}: P_{i} & \rightarrow P_{i} \\
\mathrm{w}_{i}^{H} & \mapsto \mathrm{w}_{i}^{H} & \mathrm{w}_{i}^{H} & \mapsto \mathrm{w}_{i}^{S} \\
\alpha_{i}^{+}: P_{i} & \rightarrow \mathbb{C}_{r}^{H} \otimes P_{r-2-i} & \alpha_{i}^{-}: P_{i} & \rightarrow \mathbb{C}_{-r}^{H} \otimes P_{r-2-i} . \\
\mathrm{w}_{i}^{H} & \mapsto 1 \otimes \mathrm{w}_{i-r}^{L} & \mathrm{w}_{i}^{H} & \mapsto[i] !^{-2} 1 \otimes \mathrm{w}_{i+r}^{R}
\end{aligned}
$$

Proof. By Equality (27), the space $\operatorname{Hom}_{\mathscr{C}}\left(P_{i}, P\right)$ is isomorphic to the space of dominant weight vectors of weight $i$ of $P$. Now the space of dominant vectors of $\mathbb{C}_{k r}^{H} \otimes P_{\ell}$ has dimension 4 and is generated by $1 \otimes \mathrm{w}_{\ell}^{H}$ and $1 \otimes \mathrm{w}_{\ell}^{S}$ of weight $\ell+k r, 1 \otimes \mathrm{w}_{-\ell-2}^{L}$ of weight $-\ell-2+k r$ and $1 \otimes \mathrm{w}_{2 r-\ell-2}^{R}$ of weight $(k+2) r-\ell-2$. The result then follows by analyzing for which $k, \ell$ the module $\mathbb{C}_{k r}^{H} \otimes P_{\ell}$ has dominant weight vectors of weight $i$.

Tensoring by $\mathbb{C}_{n r}^{H}$ gives canonical isomorphisms $\operatorname{Hom}_{\mathscr{C}}\left(P_{i}, \mathbb{C}_{k r}^{H} \otimes P_{\ell}\right) \cong \operatorname{Hom}_{\mathscr{C}}\left(\mathbb{C}_{n r}^{H} \otimes\right.$ $\left.P_{i}, \mathbb{C}_{(n+k) r}^{H} \otimes P_{\ell}\right)$. Then for $i \in\{0, \ldots, r-2\}$ and $j=r-2-i$, maps between indecomposable projective modules $P_{i}^{k}=\mathbb{C}_{k r}^{H} \otimes P_{i}, P_{j}^{k}=\mathbb{C}_{k r}^{H} \otimes P_{j}$ can be represented by the following periodic quiver:

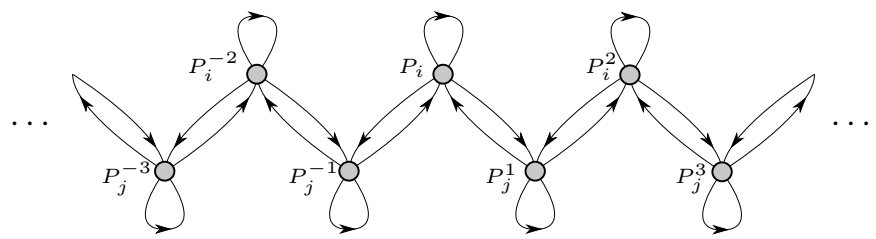

7.2. The algebras of curves. As above, let $r^{\prime}=r$ if $r$ is odd and $r^{\prime}=\frac{r}{2}$ else. Let $\sigma=\mathbb{C}_{2 r^{\prime}}^{H}$ be the one dimensional module where $E$ and $F$ act as 0 and $H$ acts as $2 r^{\prime}$. The object $\sigma \in \mathscr{C}_{\overline{0}}$ generates the group of invertible objects of $\mathscr{C}_{\overline{0}}$ which is isomorphic to $\mathbb{Z}$. For $k \in \mathbb{Z}$, we just denote by $\sigma^{k}$ the module $\mathbb{C}_{2 k r^{\prime}}^{H}$ so that $\sigma^{k} \otimes \sigma^{\ell}=\sigma^{k+\ell}$ and $\sigma^{0}=\mathbb{I}$.

A $\sigma$-invariant module is an infinite dimensional weight module $V$ with finite dimensional weight spaces and with the property that $\sigma \otimes V=V$. Then tensor product by $1 \in \sigma$ gives an action of $\mathbb{Z} \simeq\left\{\sigma^{k}: k \in \mathbb{Z}\right\}$ on $V$ denoted by $v \mapsto \sigma v$. Remark that since $V$ is infinite dimensional it is not an object of $\mathscr{C}$.

Let $\mathscr{C}^{\sigma}$ be the category whose objects are $\sigma$-invariant modules and maps from $V$ to $W$ are given by the set $\operatorname{Hom}_{\sigma}(V, W)$ of morphism of $U_{q}^{H} \mathfrak{s l}(2)$-modules that commute with the action of $\sigma$.

We study endomorphisms of the $\sigma$-invariant module

$$
\mathbb{P}=\bigoplus_{k \in \mathbb{Z}} \bigoplus_{i=0}^{r-1} \mathbb{C}_{k r}^{H} \otimes P_{i}
$$

According to the parity of weights, this module splits into two $\sigma$-invariant modules $\mathbb{P}=$ $\mathbb{P}_{\overline{0}} \oplus \mathbb{P}_{\overline{1}}$. Also for $\nu \in\{\overline{0}, \overline{1}\}, \mathbb{P}_{\nu}$ has a $\mathbb{Z}$-grading for which $\mathbb{C}_{k r}^{H} \otimes P_{i}$ is of degree $k$. In particular, if $r$ is odd, the action of $\sigma=\mathbb{C}_{2 r}^{H}$ shifts the degree by 2 whereas for $r$ even, $\sigma=\mathbb{C}_{r}^{H}$ shifts the degree by 1 . In the standard way, the $\mathbb{Z}$-grading of $\mathbb{P}_{\nu}$ turns $\operatorname{End}_{\sigma}\left(\mathbb{P}_{\nu}\right)$ into a $\mathbb{Z}$-graded algebra. We call

$$
\mathbb{A}_{\overline{0}}=\operatorname{End}_{\sigma}\left(\mathbb{P}_{\overline{0}}\right) \quad \text { and } \quad \mathbb{A}_{\overline{1}}=\operatorname{End}_{\sigma}\left(\mathbb{P}_{\overline{1}}\right)
$$


these $\mathbb{Z}$-graded algebras.

We now introduce two algebras $A$ and $B$ used to describe $\mathbb{A}_{\nu}=\operatorname{End}_{\sigma}\left(\mathbb{P}_{\nu}\right)$. Let $A$ be the algebra of graded dimension $2 s^{-1}+4+2 s$ which is the quotient of the $\mathbb{C}$-path algebra associated to the quiver

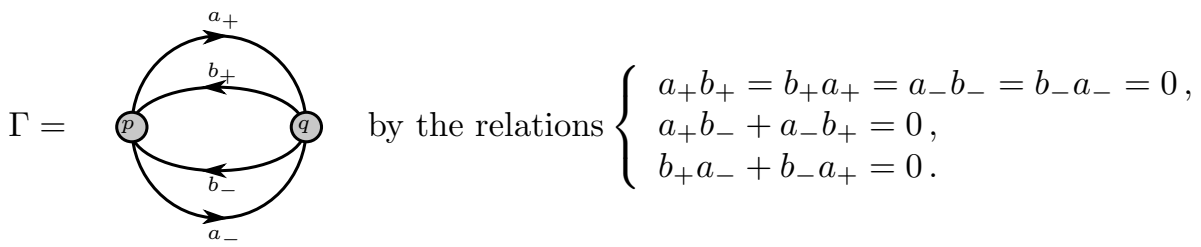

As a $\mathbb{Z}$-graded $\mathbb{C}$-vector space, $A$ is spanned in degree 1 by $\left\{a_{+}, b_{+}\right\}$, in degree -1 by $\left\{a_{-}, b_{-}\right\}$and in degree 0 by $\left\{p, q=1-p, x=b_{+} a_{-}, y=a_{+} b_{-}\right\}$.

The algebra $B$ is the quotient of $A$ obtained by identifying $p=q, a_{+}=b_{+}$and $a_{-}=b_{-}$ $\left(B\right.$ is the exterior algebra of $\mathbb{C}^{2}$ ). It is also the quotient of the $\mathbb{C}$-path algebra associated to the quiver

$$
\Gamma^{\prime}=\overbrace{a_{-}} \text {by the relations }\left\{\begin{array}{l}
a_{+} a_{+}=a_{-} a_{-}=0, \\
a_{+} a_{-}+a_{-} a_{+}=0 .
\end{array}\right.
$$

The basis of $B$ is given by $\left\{a_{-}, p=1, x=a_{+} a_{-}, a_{+}\right\}$.

Theorem 7.2. There exist isomorphisms of algebras:

$$
\begin{aligned}
& \text { If } r \in 3+2 \mathbb{N}, \mathbb{A}_{\overline{0}}=\operatorname{End}_{\sigma}\left(\mathbb{P}_{\overline{0}}\right) \simeq A^{\frac{r-1}{2}} \times \mathbb{C} \simeq \operatorname{End}_{\sigma}\left(\mathbb{P}_{\overline{1}}\right)=\mathbb{A}_{\overline{1}} . \\
& \text { If } r \in 2+4 \mathbb{N}, \mathbb{A}_{\overline{0}}=\operatorname{End}_{\sigma}\left(\mathbb{P}_{\overline{0}}\right) \simeq A^{\frac{r-2}{4}} \times B \quad \text { and } \quad \mathbb{A}_{\overline{1}}=\operatorname{End}_{\sigma}\left(\mathbb{P}_{\overline{1}}\right) \simeq A^{\frac{r-2}{4}} \times \mathbb{C} . \\
& \text { If } r \in 4+4 \mathbb{N}, \mathbb{A}_{\overline{0}}=\operatorname{End}_{\sigma}\left(\mathbb{P}_{\overline{0}}\right) \simeq A^{\frac{r}{4}} \quad \text { and } \quad \mathbb{A}_{\overline{1}}=\operatorname{End}_{\sigma}\left(\mathbb{P}_{\overline{1}}\right) \simeq A^{\frac{r-4}{4}} \times B \times \mathbb{C} .
\end{aligned}
$$

Proof. To prove this theorem we build the explicit isomorphisms. If a $\sigma$-invariant module $W$ splits as $W=\bigoplus_{k \in \mathbb{Z}} \sigma^{k} \otimes V$ for some finite dimensional weight module $V$ then the action of $\sigma$ on $W$ is free and the restriction map $\operatorname{Hom}_{\sigma}\left(W, W^{\prime}\right) \rightarrow \operatorname{Hom}_{U_{q}^{H} \mathfrak{s l}(2)}\left(V, W^{\prime}\right)$ is easily seen to be an isomorphism (here $\operatorname{Hom}_{U_{q}^{H} \mathfrak{s l}(2)}$ denotes morphisms of $U_{q}^{H} \mathfrak{s l}(2)$-modules). Using this fact, we restrict our study to the maps from $P_{i}$ to $\mathbb{P}$. For $i \leq r-2$, let $j=r-2-i$. By Proposition 7.1. the space $\operatorname{Hom}_{U_{q}^{H} \mathfrak{s l}(2)}\left(P_{i}, \mathbb{P}\right)$ is of dimension 4 generated by the morphisms determined uniquely by

$$
\begin{aligned}
I_{i}: P_{i} \rightarrow P_{i} \subset \mathbb{P} & x_{i}: P_{i} \rightarrow P_{i} \subset \mathbb{P} \\
\alpha_{i}^{+}: P_{i} & \rightarrow \mathbb{C}_{r}^{H} \otimes P_{j} \subset \mathbb{P} \quad \alpha_{i}^{-}: P_{i} \rightarrow \mathbb{C}_{-r}^{H} \otimes P_{j} \subset \mathbb{P}
\end{aligned}
$$

These maps extend to maps of $\operatorname{End}_{\sigma}(\mathbb{P})$ on factors $\mathbb{C}_{k r}^{H} \otimes P_{i}$ by tensoring them by the identity of $\mathbb{C}_{k r}^{H}$ and we extend them by 0 on the other factors. We use the same name for these extended maps of $\operatorname{End}_{\sigma}(\mathbb{P})$. The composition of these maps is computed by looking at the image of the dominant vector $\mathrm{w}_{i}^{H} \in P_{i}$. One easily gets

$$
\alpha_{j}^{+} \circ \alpha_{i}^{+}=0=\alpha_{j}^{-} \circ \alpha_{i}^{-} .
$$


Now we use that if $v \in V$ is a weight vector then in $\mathbb{C}_{ \pm r}^{H} \otimes V$ one has $E .(1 \otimes v)=1 \otimes(E . v)$ and $F .(1 \otimes v)=1 \otimes(-F . v)$ to compute:

$$
\begin{aligned}
& P_{i} \stackrel{\alpha_{i}^{+}}{\longrightarrow} \quad \mathbb{C}_{r}^{H} \otimes P_{j} \quad \stackrel{\alpha_{j}^{-}}{\longrightarrow} P_{i} \\
& \mathrm{w}_{i}^{H} \longmapsto 1 \otimes \mathrm{w}_{i-r}^{L}=(-F)^{j+1}\left(1 \otimes \mathrm{w}_{j}^{H}\right) \longmapsto[j] !^{-2}(-F)^{j+1} \mathrm{w}_{j+r}^{R}
\end{aligned}
$$

As $F^{j+1} \mathrm{w}_{j+r}^{R}=\prod_{k=1}^{j}\left(-\gamma_{j, j-k+1}\right) \mathrm{w}_{i}^{S}=(-1)^{j}[j] !^{2} \mathrm{w}_{i}^{S}$, we get

$$
\alpha_{j}^{-} \circ \alpha_{i}^{+}=-x_{i}
$$

Similarly,

$$
\begin{aligned}
& P_{i} \stackrel{\alpha_{i}^{-}}{\longrightarrow} \quad \mathbb{C}_{-r}^{H} \otimes P_{j} \quad \stackrel{\alpha_{j}^{+}}{\longrightarrow} P_{i} \\
& \mathrm{w}_{i}^{H} \longmapsto[i] !^{-2} 1 \otimes \mathrm{w}_{i+r}^{R}=[i] !^{-2} E^{i+1}\left(1 \otimes \mathrm{w}_{j}^{H}\right) \longmapsto[i] !^{-2} E^{i+1} \mathrm{w}_{j-r}^{L}
\end{aligned}
$$

And as $E^{i+1} \mathrm{w}_{j-r}^{L}=\prod_{k=1}^{i}\left(\gamma_{i, k}\right) \mathrm{w}_{i}^{S}=[i] !^{2} \mathbf{w}_{i}^{S}$, we get

$$
\alpha_{j}^{+} \circ \alpha_{i}^{-}=x_{i} \text {. }
$$

We now explicit the isomorphism of Theorem 7.2. First remark that the maps of $\operatorname{End}_{\sigma}(\mathbb{P})$ commute with $K^{r}$ thus they restrict to maps of $\operatorname{End}_{\sigma}\left(\mathbb{P}_{\nu}\right)$ for $\nu \in\{\overline{0}, \overline{1}\}$. Next the decomposition of endomorphism algebras in Theorem 7.2 follows from the fact that these endomorphisms respect the characteristic spaces of the Casimir element $C$ whose minimal polynomial is given in Proposition 4.1 . For $i \in\{0 \cdots r-1\}$, let $c_{i}=\frac{q^{i+1}+q^{-i-1}}{\{1\}^{2}}=$ $-\frac{q^{j+1}+q^{-j-1}}{\{1\}^{2}}$ be the scalar by which $C$ acts on the simple module $S_{i}$. The action of $C$ on $\sigma \otimes S_{i}$ and on $S_{i}$ are the same if $r$ is odd, but they are opposite if $r$ is even.

Let $\nu \in\{0,1\}$. For $i \in 2 \mathbb{N}+\nu, i \leq r^{\prime}-2$, the kernel of $\left(C^{2}-c_{i}^{2}\right)^{2}$ on $\mathbb{P}_{\nu}$ is $V=$ $\bigoplus_{k \in \mathbb{Z}} \sigma^{k} \otimes\left(P_{i} \oplus Q_{j}\right)$ where $Q_{j}$ is $P_{j}$ if $r$ even and $Q_{j}=\mathbb{C}_{r}^{H} \otimes P_{j}$ if $r$ is odd. Then an isomorphism $A \stackrel{\sim}{\rightarrow} \operatorname{End}_{\sigma}(V)$ is given by

$$
\begin{array}{rlrlrl}
p & \mapsto I_{i} & x & \mapsto x_{i} & a^{+} \mapsto \alpha_{i}^{+} & a^{-} \mapsto \alpha_{i}^{-} \\
q \mapsto I_{j} & y & \mapsto x_{j} & b^{+} \mapsto \alpha_{j}^{+} & b^{-} \mapsto \alpha_{j}^{-}
\end{array}
$$

Now if $r$ is even, let $i=\frac{r-2}{2}=r-2-i$ and $\nu=i \bmod 2$. Then $c_{i}=0$ and the kernel of $C^{2}$ on $\mathbb{P}_{\nu}$ is then $V=\bigoplus_{k \in \mathbb{Z}} \sigma^{k} \otimes P_{i}$. Then an isomorphism $B \stackrel{\sim}{\rightarrow} \operatorname{End}_{\sigma}(V)$ is given by

$$
p \mapsto I_{i} \quad x \mapsto x_{i} \quad a^{+} \mapsto \alpha_{i}^{+} \quad a^{-} \mapsto \alpha_{i}^{-}
$$

Finally the remaining $\mathbb{C}$ factors in Theorem 7.2 correspond to the eigenspace of $C$ associated to the simple eigenvalue $c_{r-1}$.

In the paper 3, the concepts of Coend, trace and the Hochschild-Mitchell homology in a linear category are related. In 4, a graded TQFT is defined for manifolds equipped with a 1 -cohomology class with value in $\mathbb{C} / 2 \mathbb{Z}$. The algebras $\mathbb{A}_{\nu}$ would naturally be associated to a curve $\gamma$ with cohomology class $\omega$ such that $\omega([\gamma])=\nu$. Then the graded vector space $\operatorname{Tr}\left(\mathbb{A}_{\nu}\right)=\left(\mathbb{A}_{\nu}\right)_{/ f g=g f}$ maps surjectively onto the TQFT space of the torus $\gamma \times S^{1}$ with cohomology class $\omega$ such that $\omega([\gamma \times *])=\nu$ and $\omega\left(\left[* \times S^{1}\right]\right)=0$. Here we define a graded version of the trace of $\mathbb{A}_{\nu}$ that surjects on the TQFT space of the torus $\gamma \times S^{1}$ with cohomology class $\omega$ such that $\omega([\gamma \times *])=\nu$ and $\omega\left(\left[* \times S^{1}\right]\right)=\beta$ for any $\beta \in \mathbb{C} / 2 \mathbb{Z}$ (instead of $\beta$, we use $z=q^{2 r^{\prime} \beta}$ ).

Let $z \in \mathbb{C}^{*}, \mathcal{A}$ be a $\mathbb{Z}$-graded $\mathbb{C}$-algebra. If $f, g$ are homogenous elements of degree $|f|,|g| \in \mathbb{Z}$, let $[f, g]_{z}=f g-z^{|f|} g f$. Define the $\mathbb{Z}$-graded module

$$
\operatorname{Tr}^{z}(\mathcal{A})=\mathcal{A} /[\mathcal{A}, \mathcal{A}]_{z}
$$


Similarly, if $\mathcal{A}$ is considered as a super algebra, the bracket is replaced by $[f, g]_{z}^{-}=$ $f g-(-1)^{|f||g|} z^{|f|} g f$ and we define the $\mathbb{Z}$-graded super module

$$
\operatorname{STr}^{z}(\mathcal{A})=\mathcal{A}_{/[\mathcal{A}, \mathcal{A}]_{z}^{-}}
$$

Proposition 7.3. Recall the algebras $A$ and $B$ above. Then

(1) If $z \in \mathbb{C}^{*} \backslash\{ \pm 1\}, \operatorname{Tr}^{z}(A) \simeq \mathbb{C}^{2} \simeq \operatorname{STr}^{z}(A)$.

(2) $\operatorname{Tr}^{ \pm 1}(A) \simeq \mathbb{C}^{3} \simeq \operatorname{STr}^{ \pm 1}(A)$.

(3) If $z \neq 1$ then $\operatorname{STr}^{z}(B) \simeq \mathbb{C}$ and $\operatorname{STr}^{1}(B) \simeq B$.

Here the spaces $\mathbb{C}^{2}$ and $\mathbb{C}^{3}$ are concentrated in degree 0 . As a consequence, we have the following graded dimensions:

(1) If $r \in 2 \mathbb{Z}+1$ and $z \neq \pm 1$, then

$\operatorname{dim}_{s}\left(\operatorname{Tr}^{z}\left(\mathbb{A}_{\overline{0}}\right)\right)=\operatorname{dim}_{s}\left(\operatorname{Tr}^{z}\left(\mathbb{A}_{\overline{1}}\right)\right)=r \quad$ and $\quad \operatorname{dim}_{s}\left(\operatorname{Tr}^{ \pm 1}\left(\mathbb{A}_{\overline{0}}\right)\right)=\operatorname{dim}_{s}\left(\operatorname{Tr}^{ \pm 1}\left(\mathbb{A}_{\overline{1}}\right)\right)=\frac{3 r-1}{2}$.

(2) If $r \in 4 \mathbb{Z}+2$ and $z \neq \pm 1$, then

$$
\begin{gathered}
\operatorname{dim}_{s}\left(\mathrm{STr}^{z}\left(\mathbb{A}_{\overline{0}}\right)\right)=\operatorname{dim}_{s}\left(\mathrm{STr}^{z}\left(\mathbb{A}_{\overline{1}}\right)\right)=\frac{r}{2}, \\
\operatorname{dim}_{s}\left(\mathrm{STr}^{ \pm 1}\left(\mathbb{A}_{\overline{1}}\right)\right)=\operatorname{dim}_{s}\left(\mathrm{STr}^{-1}\left(\mathbb{A}_{\overline{0}}\right)\right)=\frac{3 r-2}{4} \text { and } \\
\operatorname{dim}_{s}\left(\mathrm{STr}^{1}\left(\mathbb{A}_{\overline{0}}\right)\right)=s^{-1}+\frac{3 r+2}{4}+s
\end{gathered}
$$

where $\operatorname{dim}_{s}$ is the sum for $k \in \mathbb{Z}$ of $s^{k}$ times the dimension of the degree $k$ subspace.

Proof. Let $\varepsilon= \pm 1$. First remark that for any elements $f, g$ of the algebra, $[f, g]_{z}^{\varepsilon}+$ ${ }^{|f| \cdot|g|} z^{|f|}[g, f]_{z}^{\varepsilon}=\left(1-z^{|f|+|g|}\right) f g$. Hence if $z^{|f|+|g|} \neq 1$ then $f g=0$ in the quotient, else $[g, f]_{z}^{\varepsilon}$ and $[f, g]_{z}^{\varepsilon}$ are proportional. Finally for $g=1$, one gets that a map $f$ vanishes in the quotient unless $z^{|f|}=1$. Then the relations in $A$ implies that $[A, A]_{z}^{\varepsilon}$ is generated by the following elements

- $\left[a_{ \pm}, p\right]_{z}^{\varepsilon}=a_{ \pm}$

- $\left[b_{ \pm}, q\right]_{z}^{\varepsilon}=b_{ \pm}$

- $\left[b_{+}, a_{-}\right]_{z}^{\varepsilon}=b_{+} a_{-}-\varepsilon z a_{-} b_{+}=x+\varepsilon z y$

- $\left[a_{+}, b_{-}\right]_{z}^{\varepsilon}=a_{+} b_{-}-\varepsilon z b_{-} a_{+}=y+\varepsilon z x$

If $z^{2} \neq 1$ then $x=y=0$ in the quotient, and if $z= \pm 1$, then $[A, A]_{z}^{\varepsilon}$ is generated in degree 0 by $x+\varepsilon z y$.

Similarly for $z \neq 1,[B, B]_{z}^{-}$is generated by $a_{+}, a_{-}$and the element

$$
\left[a_{+}, a_{-}\right]_{z}^{-}=a_{+} a_{-}+z a_{-} a_{+}=(1-z) x .
$$

On the other hand, for $z=1$ we have $[B, B]_{1}^{-}=0$.

For the last statements, we use $\operatorname{Tr}^{z}\left(\mathcal{A} \times \mathcal{A}^{\prime}\right)=\operatorname{Tr}^{z}(\mathcal{A}) \oplus \operatorname{Tr}^{z}\left(\mathcal{A}^{\prime}\right)$ and $\operatorname{STr}^{z}\left(\mathcal{A} \times \mathcal{A}^{\prime}\right)=$ $\mathrm{STr}^{z}(\mathcal{A}) \oplus \operatorname{STr}^{z}\left(\mathcal{A}^{\prime}\right)$.

\section{Decomposition of tensor PRoducts}

We recall the different notations for the simple self-dual projective module:

$$
P_{r-1}=V_{0}=S_{r-1} \text {. }
$$

From Proposition 6.2 any projective indecomposable module of $\mathscr{C}_{\overline{0}} \cup \mathscr{C}_{\overline{1}}$ is an element of the set $\left\{P_{i} \otimes \mathbb{C}_{k r}^{H}, i \in\{0,1, \ldots r-1\}, k \in \mathbb{Z}\right\}$. Let us recall their characters

$$
\chi\left(P_{i} \otimes \mathbb{C}_{k r}^{H}\right)=X^{k r}[r]_{X}\left(X^{r-i-1}+X^{-r+i+1}\right) \quad \text { and } \quad \chi\left(V_{0} \otimes \mathbb{C}_{k r}^{H}\right)=X^{r k}[r]_{X}
$$


where $i \in\{0,1, \ldots r-2\}$. Observe now that these characters are linearly independent in $\mathbb{Z}\left[X^{ \pm 1}\right]$ and form a basis of an ideal of polynomials which are divisible by $[r]_{X}$ (but not of the whole ideal generated by $[r]_{X}$ ).

As a consequence to decompose a projective module $P$ in direct sum of projective indecomposable ones, it is sufficient to decompose $\chi(P)$ as

$$
\chi(P)=\sum_{i=0}^{r-1} \sum_{k_{i} \in \mathbb{Z}} n_{i, k_{i}} \chi\left(\mathbb{C}_{k_{i} r}^{H} \otimes P_{i}\right) .
$$

In the following, we write

$$
\sum_{\substack{k=m \\ \text { by } 2}}^{n} \text { and } \bigoplus_{\substack{k=m \\ \text { by } 2}}^{n}
$$

for the sums where $k$ is $k \leq n$ and varies in the set $m+2 \mathbb{N}$. Similarly, we write

$$
\sum_{\substack{k=n \\ \text { by }-2}}^{m} \text { and } \bigoplus_{\substack{k=n \\ \text { by }-2}}^{m}
$$

for the sums where $k \geq m$ and varies in the set $n-2 \mathbb{N}$.

Lemma 8.1 (Decomposition of tensor products $V_{0} \otimes S_{i}$ ). Let $0 \leq i \leq r-1$. Then

$$
V_{0} \otimes S_{i}=\bigoplus_{\substack{r-1-i \\ b y 2}}^{r-1} P_{k}
$$

Proof. If $i$ is odd it holds :

$$
\chi\left(V_{0} \otimes S_{i}\right)=[r]_{X}[i+1]_{X}=[r]_{X} \sum_{\substack{j=i \\ \text { by }-2}}^{1}\left(X^{j}+X^{-j}\right)=\sum_{\substack{j=i \\ \text { by }-2}}^{0} \chi\left(P_{r-1-j}\right) .
$$

If $i$ is even it holds :

$$
\chi\left(V_{0} \otimes S_{i}\right)=[r]_{X}[i+1]_{X}=[r]_{X}\left(1+\sum_{\substack{j=i \\ \text { by }-2}}^{2}\left(X^{j}+X^{-j}\right)\right)=\sum_{\substack{j=i \\ \text { by }-2}}^{0} \chi\left(P_{r-1-j}\right) .
$$

Proposition 8.2 (The decomposition of the tensor products $P_{i} \otimes S_{j}$ ). Let $0 \leq i \leq r-2$ and $0 \leq j \leq r-1$. It holds:

$$
P_{i} \otimes S_{j}=\left(\bigoplus_{\substack{k=|i-j| \\ b y 2}}^{\min (i+j, r-1)} P_{k}\right) \oplus\left(\bigoplus_{\substack{k=2-i-j \\ b y 2}}^{r-1} P_{k}\right) \oplus\left(\bigoplus_{\substack{r+i-j \\ b y 2}}^{r-1} P_{k} \otimes\left(\mathbb{C}_{r}^{H} \oplus \mathbb{C}_{-r}^{H}\right)\right)
$$

where the sums are meant to be empty if the lower bound is bigger than the upper bound.

Proof. It holds :

$$
\chi\left(P_{i} \otimes S_{j}\right)=[r]_{X}\left(X^{r-i-1}+X^{-r+i+1}\right)[j+1]_{X}=[r]_{X}\left([r-i+j]_{X}-[r-i-j-2]_{X}\right) .
$$


Recall that $[-n]=-[n]$. We denote the parity of $r-1-i+j$ and $r-3-i-j$ by $p \in\{0,1\}$ (note they coincide). If $i>j$ and $i+j \leq r-2$ we have :

$\chi\left(P_{i} \otimes S_{j}\right)=[r]_{X}\left(\sum_{\substack{l=-i-j-1 \\ \text { by } 2}}^{r-i+j-1}\left(X^{l}+X^{-l}\right)\right)=\sum_{\substack{l=r-i-j-1 \\ \text { by } 2}}^{r-i+j-1} \chi\left(P_{r-1-l}\right)=\sum_{\substack{k=i-j \\ \text { by } 2}}^{i+j} \chi\left(P_{k}\right)$.

If $i \geq j$ and $i+j \geq r-1$ and $p=1$ we have :

$$
\begin{aligned}
& \chi\left(P_{i} \otimes S_{j}\right)=[r]_{X}\left(\sum_{\substack{l=p \\
\text { by } 2}}^{r-i+j-1}\left(X^{l}+X^{-l}\right)+\sum_{\substack{l=p \\
\text { by } 2}}^{i+j-r+1}\left(X^{l}+X^{-l}\right)\right)= \\
& =\sum_{\substack{l=p \\
\text { by } 2}}^{r-i+j-1} \chi\left(P_{r-1-l}\right)+\sum_{\substack{l=p \\
\text { by } 2}}^{i+j-r+1} \chi\left(P_{r-1-l}\right)=\sum_{\substack{k=i-j \\
\text { by } 2}}^{r-1-p} \chi\left(P_{k}\right)+\sum_{\substack{k=2-i-j \\
\text { by } 2}}^{r-1-p} \chi\left(P_{k}\right) \text {. }
\end{aligned}
$$

Note a similar calculation gives the result above in the case $p=0$ (just pay attention to the fact that if $p=0$ the terms $X^{0}+X^{-0}$ should be replaced by $\left.X^{0}\right)$.

Let us now suppose that $j>i$ and $i+j \leq r-2$ and let $q \in\{0,1\}$ be the parity of $j-i-1$. Then, if $q=1$ (as above, a similar calculation proves the same final formula if $q=0)$ it holds :

$$
\begin{array}{r}
\chi\left(P_{i} \otimes S_{j}\right)=[r]_{X}\left(\sum_{\substack{l=r-j+i+1 \\
\text { by } 2}}^{r-i+j-1}\left(X^{l}+X^{-l}\right)+\sum_{\substack{l=r-i-j-1 \\
\text { by } 2}}^{r-j+i-1}\left(X^{l}+X^{-l}\right)\right) \\
\left.[r]_{X}\left(X^{r}+X^{-r}\right) \sum_{\substack{h=q \\
\text { by } 2}}^{j-i-1}\left(X^{h}+X^{-h}\right)+\sum_{\substack{l=r-i-j-1 \\
\text { by } 2}}^{r-j+i-1}\left(X^{l}+X^{-l}\right)\right) \\
=\left(X^{r}+X^{-r}\right) \sum_{\substack{k=-j+i \\
\text { by } 2}}^{r-1-q} \chi\left(P_{k}\right)+\sum_{\substack{k=j-i \\
\text { by } 2}}^{i+j} \chi\left(P_{k}\right) .
\end{array}
$$

Finally suppose that $j>i$ and $i+j \geq r-1$ and as before let $p \in\{0,1\}$ be the parity of $r-i+j-1$. If $p=1$ (and as above if $p=0$ or $q=0$ modify the calculation by replacing 
the terms $X^{0}+X^{-0}$ by $X^{0}$, still getting the same final result):

$$
\begin{aligned}
& \chi\left(P_{i} \otimes S_{j}\right)=[r]_{X}\left(\sum_{\substack{l=p \\
\text { by } 2}}^{r-i+j-1}\left(X^{l}+X^{-l}\right)+\sum_{\substack{l=p \\
\text { by } 2}}^{i+j+1-r}\left(X^{l}+X^{-l}\right)\right)= \\
& {[r]_{X}\left(\sum_{\substack{r-j+i+1 \\
\text { by } 2}}^{r+j-i-1} X^{h}+\sum_{\substack{h=j+i+1 \\
\text { by } 2}}^{r+j-i-1} X^{-h}+\sum_{\substack{h=p \\
\text { by } 2}}^{r-j+i-1}\left(X^{h}+X^{-h}\right)+\sum_{\substack{l=p \\
\text { by } 2}}^{i+j+1-r}\left(X^{l}+X^{-l}\right)\right)=} \\
& =[r]_{X}\left(\left(X^{r}+X^{-r}\right) \sum_{\substack{s=q \\
\text { by } 2}}^{j-i-1}\left(X^{s}+X^{-s}\right)+\sum_{\substack{h=p \\
\text { by } 2}}^{r-j+i-1}\left(X^{h}+X^{-h}\right)+\sum_{\substack{l=p \\
\text { by } 2}}^{i+j+1-r}\left(X^{l}+X^{-l}\right)\right)= \\
& =\left(X^{r}+X^{-r}\right) \sum_{\substack{r-j+i \\
\text { by } 2}}^{r-1-q} \chi\left(P_{k}\right)+\sum_{\substack{k=j-i \\
\text { by } 2}}^{r-1-p} \chi\left(P_{k}\right)+\sum_{\substack{2 r-2-i-j \\
\text { by } 2}}^{r-1-p} \chi\left(P_{k}\right) \text {. }
\end{aligned}
$$

To summarize the above computations, let $p, q \in\{0,1\}$ be the parities of $r+j-i-1$ and of $j-i-1$, respectively. It holds:

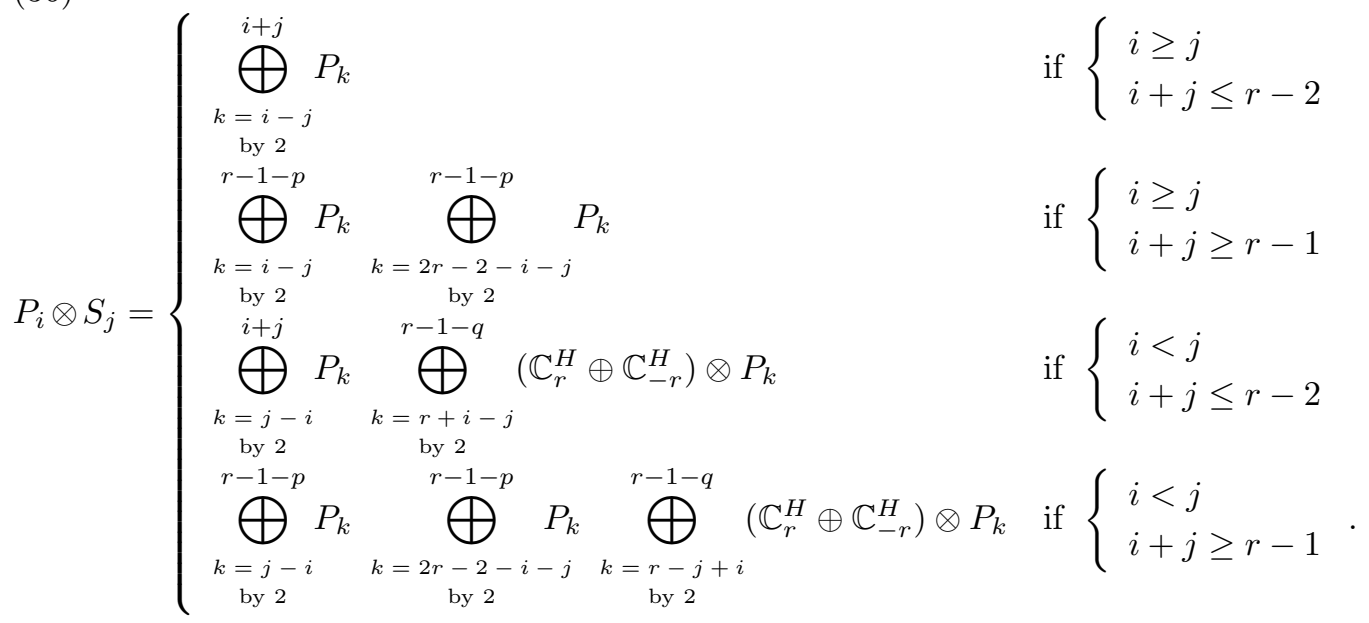

This is equivalent to the statement of the proposition.

Let us now remark that for each $i \in\{0,1, \ldots r-2\}$,

$$
\chi\left(P_{i}\right)=2 \chi\left(S_{i}\right)+\left(\chi\left(\mathbb{C}_{r}^{H}\right)+\chi\left(\mathbb{C}_{-r}^{H}\right)\right) \chi\left(S_{r-2-i}\right) .
$$

This, together with Proposition 8.2 and the fact that the modules $P_{i}$ are projective allow us to compute the full tensor decomposition of $P_{i} \otimes P_{j}$ :

Corollary 8.3 (The tensor decomposition of $P_{i} \otimes P_{j}$ ). For each $i, j \in\{0,1, \ldots r-2\}$ we have

$$
P_{i} \otimes P_{j}=\left(\left(\mathbb{C}_{r}^{H} \oplus \mathbb{C}_{-r}^{H}\right) \otimes\left(P_{i} \otimes S_{r-2-j}\right)\right) \bigoplus 2\left(P_{i} \otimes S_{j}\right)
$$


and so

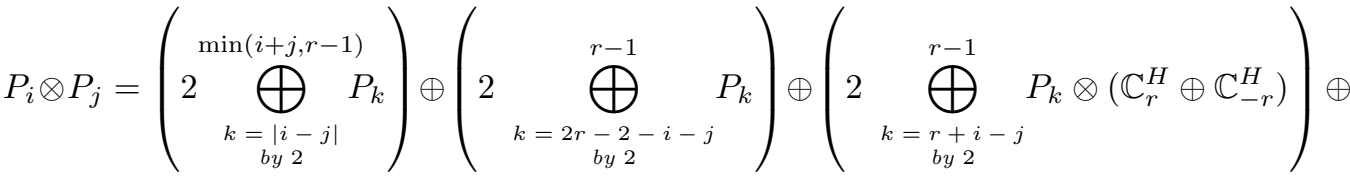

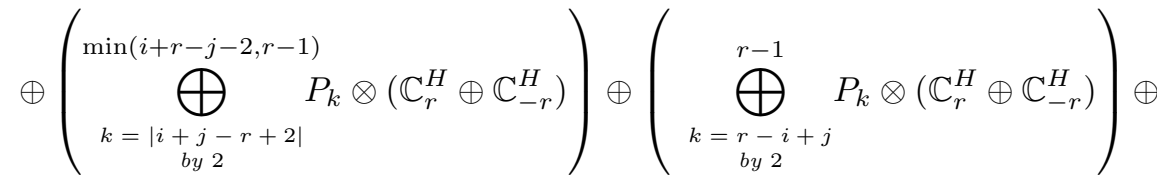

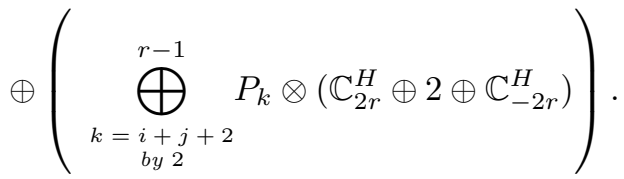

Similarly $V_{0} \otimes P_{j}=\left(\left(\mathbb{C}_{r}^{H} \oplus \mathbb{C}_{-r}^{H}\right) \otimes\left(V_{0} \otimes S_{r-2-j}\right)\right) \bigoplus 2\left(V_{0} \otimes S_{j}\right)$, and so

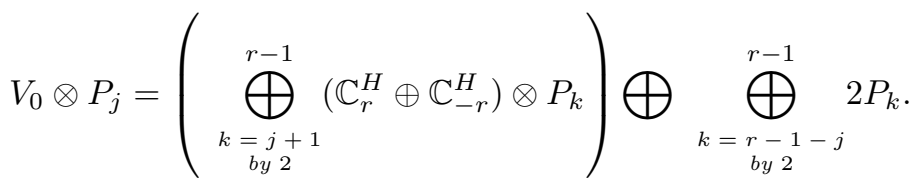

Proposition 8.4. Let $i, j \in\{0 . . r-1\}$. If $i+j \leq r-1$, then

$$
S_{i} \otimes S_{j}=\bigoplus_{\substack{k=|i-j| \\ b y 2}}^{i+j} S_{k}
$$

If $i+j \geq r$ then

$$
S_{i} \otimes S_{j}=\bigoplus_{\substack{k=|i-j| \\ b y 2}}^{2 r-4-i-j} S_{k} \oplus \bigoplus_{k=2 r-2-i-j}^{r-1} P_{k} .
$$

In particular, semi-simple and projective modules of $\mathscr{C}$ form a full sub-tensor category.

Proof. The proof is by induction on $i$ using that for $j \in\{1 \cdots r-2\}, S_{1} \otimes S_{j}=S_{j-1} \oplus S_{j+1}$ and that $S_{r-1}$ is projective. The induction is given by using

$$
S_{1} \otimes S_{i} \otimes S_{j}=\left(S_{i+1} \otimes S_{j}\right) \oplus\left(S_{i-1} \otimes S_{j}\right)
$$

To see the last point, remark that the tensor product of two simple modules is a direct sum of a semi-simple module direct sum a projective module. Thus, the full subcategory formed by semi-simple and projective modules is stable by tensor product.

\section{Multiplicity modules}

Here we summarize some known facts about multiplicity modules. The one dimensional Hom spaces $\operatorname{Hom}_{\mathscr{C}}\left(\mathbb{C}, V_{\alpha} \otimes V_{-\alpha}\right)$ and $\operatorname{Hom}_{\mathscr{C}}\left(\mathbb{C}, V_{\alpha} \otimes V_{\beta} \otimes V_{\gamma}\right)$ for $\alpha+\beta+\gamma \in\{-(r-1),-(r-$ $3), \ldots, r-1\}$ can be equipped with nice basis. By a nice basis of $\operatorname{Hom}_{\mathscr{C}}\left(\mathbb{C}, V_{\alpha_{1}} \otimes \cdots \otimes V_{\alpha_{n}}\right)$ we mean a set of basis of these spaces spaces such that

(1) it depends analytically of the parameters $\alpha_{i} \in \ddot{\mathbb{C}}$ (here we identify $V_{\alpha}$ with $\mathbb{C}^{r}=$ $\bigoplus_{i}$ C. $v_{i}$ as in Equation (15) and 
(2) the set of basis is globally permuted by the pivotal isomorphism

$$
\operatorname{Hom}_{\mathscr{C}}\left(\mathbb{C}, V_{\alpha_{1}} \otimes \cdots \otimes V_{\alpha_{n}}\right) \stackrel{\sim}{\longrightarrow} \operatorname{Hom}_{\mathscr{C}}\left(\mathbb{C}, V_{\alpha_{2}} \otimes \cdots \otimes V_{\alpha_{n}} \otimes V_{\alpha_{1}}\right) .
$$

The existence of a nice basis has been checked in [22] for $r$ odd and in [10] for any $r$ but using a different normalizations. These basis are used in [25, 22, 10, 8, 4, to produce numerical invariant of $\ddot{\mathbb{C}}$-colored framed trivalent graphs embedded in $S^{3}$, and numerical $6 j$-symbols.

The basis of $\operatorname{Hom}_{\mathscr{C}}\left(\mathbb{C}, V_{\alpha} \otimes V_{-\alpha}\right)$ induce isomorphisms $w_{\alpha}: V_{\alpha} \rightarrow V_{-\alpha}^{*}$ forming what is called a basic data (see 25]). Using these isomorphisms and the modified trace one gets a duality

$$
\operatorname{Hom}_{\mathscr{C}}\left(\mathbb{C}, V_{\alpha} \otimes V_{\beta} \otimes V_{\gamma}\right) \otimes \operatorname{Hom}_{\mathscr{C}}\left(\mathbb{C}, V_{-\gamma} \otimes V_{-\beta} \otimes V_{-\alpha}\right) \rightarrow \mathbb{C}
$$

for which the basis are dual to each other.

The version $U$ of quantum $\mathfrak{s l}(2)$ used in [10] is slightly different from $U_{q} \mathfrak{s l}(2)$. To differentiate these algebras, let us call $K_{U}, E_{U}, F_{U} \in U$ the generators, then there is a morphism of Hopf algebras $U_{q} \mathfrak{s l}(2) \rightarrow U$ given by sending

$$
K, E, F \text { to respectively } K_{U}^{2}, K_{U} E_{U}, F_{U} K_{U}^{-1} \text {. }
$$

through this morphism, the module $V^{a}$ of [10] can be identified with the module $V_{\alpha}$ where $\alpha=2 a-r+1$. Then the nice basis are given in [10] by computing some Clebsch-Gordan coefficients.

Different nice basis were computed in 22. They were computed recursively using the morphisms $X: V_{\alpha} \otimes V_{\beta} \rightarrow V_{\alpha+1} \otimes V_{\beta+1}$ given by

$$
X: v_{i} \otimes v_{j} \mapsto q^{\beta+i-j-1}\{\alpha-i\} v_{i} \otimes v_{j+1}+q^{-1}\{\beta-j\} v_{i+1} \otimes v_{j} .
$$

More than analytic in the parameters $\alpha_{i}$, they are given by Laurent polynomials in $q^{\alpha_{i}}$. But the work of [22] only consider odd values of $r$.

\section{OdD ROOTS OF UNITY}

In this section we briefly discuss the quantum group of Subsection 2.2 when $r \in 2 \mathbb{N}+3$ is odd and $q=\mathrm{e}^{\frac{2 \pi \sqrt{-1}}{r}}$ is a $r^{t h}$-root of unity. The reason why this case is not treated with the other are historic, technical, and due to the belief than topological applications won't differ from the case $q=\mathrm{e}^{\frac{\pi \sqrt{-1}}{r}}$.

Here the simple modules are

(1) the dimension $r$ typical modules $\left\{V_{\alpha}: \alpha \in \ddot{\mathbb{C}}\right\}$ where now $\ddot{\mathbb{C}}=\left(\mathbb{C} \backslash \frac{1}{2} \mathbb{Z}\right) \cup \frac{r}{2} \mathbb{Z}$,

(2) the dimension 1 invertible modules $\left\{\mathbb{C}_{k \frac{r}{2}}^{H}: k \in \mathbb{Z}\right\}$, and

(3) the simple modules of dimension less than $r:\left\{S_{i} \otimes \mathbb{C}_{k \frac{r}{2}}^{H}: 0<i<r, k \in \mathbb{Z}\right\}$, where the highest weight of $S_{i}$ is $i$.

One difference between the odd/even case is that Ohtsuki in 29 does not treat the case discussed in this subsection. In any case, when $r \in 2 \mathbb{N}+3$ the category is still pivotal with the same pivot given by $K^{r-1}$. The fact that the formula (5) still defines a braiding on the category $\mathscr{C}$ is proven in [23, section 5.8]. The computation of Ohtsuki for the associated twist has never been completed in this case. Still in [23] we show that a full subcategory of $\mathscr{C}$ that contain typical modules and self-dual modules is ribbon.

If $g \in \mathbb{C} / 2 \mathbb{Z} \backslash\left(\frac{1}{2} \mathbb{Z}\right) / 2 \mathbb{Z}$ then $\mathscr{C}_{g}$ is semi-simple and $\mathscr{C}_{g} \subset$ Proj. Typical modules are projective and there exists a unique trace on Proj up to a scalar. Its associated modified dimension is given by formula 18 . 
A nice basis for the multiplicity modules $\operatorname{Hom}\left(\mathbb{C}, V_{\alpha} \otimes V_{\beta} \otimes V_{\gamma}\right)$ is missing in the literature, and the $6 j$-symbols have not been computed in this case (they have been computed when $q$ is a 2 times odd root of unity in [22] and for any even root of unity in [10] with a different normalization).

In 25, 23, 8, the authors construct topological invariants of dimension 3 using algebraic data. The case treated with most attention is that of quantum $\mathfrak{s l}(2)$ when $q$ is a root of unity of order $2 r$ but the case we have discussed in this section is also considered as an example all together with the quantum groups associated to the other simple Lie algebras (also at odd root of unity).

\section{REFERENCES}

1. D. Adamović and A. Milas - Logarithmic intertwining operators and $\mathscr{W}(2,2 p-1)$ algebras. J. Math. Phys. 48 (2007), no. 7, 073503, 20 p.

2. Y. Akutsu, T. Deguchi, and T. Ohtsuki - Invariants of colored links. J. Knot Theory Ramifications 1 (1992), no. 2, 161-184.

3. A. Beliakova, K. Habiro, A. Lauda, M. Živković - Trace decategorification of categorified quantum $\operatorname{sl}(2)$, arXiv:1404.1806.

4. C. Blanchet, F. Costantino, N. Geer, and B. Patureau-Mirand - Non semi-simple TQFTs, Reidemeister torsion and Kashaev's invariants. Preprint, arXiv:1404.7289.

5. P. Bushlanov, A. Gainutdinov and I. Tipunin - Kazhdan-Lusztig equivalence and fusion of Kac modules in Virasoro logarithmic models, Nuclear Phys. B 862 (2012), no. 1, 232-269.

6. P. Bushlanov, B. Feigin, A. Gainutdinov, and I. Tipunin - Lusztig limit of quantum $\mathrm{sl}(2)$ at root of unity and fusion of $(1, p)$ Virasoro logarithmic minimal models. Nuclear Phys. B 818 (2009), no. 3, 179-195.

7. V. Chari, A. Pressley - A guide to quantum groups, Cambridge University Press, Cambridge, 1994.

8. F. Costantino, N. Geer and B. Patureau-Mirand - Quantum invariants of 3-manifolds via link surgery presentations and non-semi-simple categories. J Topology (2014) doi:10.1112/jtopol/jtu006, arXiv: 1202.3553

9. F. Costantino, N. Geer, B. Patureau-Mirand - Relations between Witten-Reshetikhin-Turaev invariants and non-semi-simple $\mathfrak{s l}(2)$ 3-manifold invariants, preprint arXiv:1310.2735 (2013).

10. F. Costantino, J. Murakami - On $S L(2, \mathbb{C})$ quantum $6 j$-symbols and its relation to the hyperbolic volume. Quantum Topology 4 (2013), no. 3, 303-351.

11. T. Creutzig and A. Milas - False Theta Functions and the Verlinde formula, Adv. Math. 262 (2014), $520-545$.

12. T. Creutzig, D. Ridout and S. Wood - Coset Constructions of Logarithmic (1,p) Models. Lett. Math. Phys. 104 (2014), no. 5, 553-583.

13. C. De Concini, V.G. Kac - Representations of quantum groups at roots of 1 . In Operator algebras, unitary representations, enveloping algebras, and invariant theory. (Paris, 1989), 471-506, Progr. Math., 92, Birkhauser Boston, 1990.

14. C. De Concini, V.G. Kac, C. Procesi - Quantum coadjoint action. J. Amer. Math. Soc. 5 (1992), no. $1,151-189$.

15. C. De Concini, V.G. Kac, C. Procesi - Some remarkable degenerations of quantum groups. Comm. Math. Phys. 157 (1993), no. 2, 405-427.

16. C. De Concini, C. Procesi, N. Reshetikhin, M. Rosso - Hopf algebras with trace and representations. Invent. Math. 161 (2005), no. 1, 1-44.

17. B. Feigin, A. Gainutdinov, A. Semikhatov and I. Tipunin - Modular group representations and fusion in logarithmic conformal field theories and in the quantum group center. Comm. Math. Phys. 265 (2006), no. 1, 47-93.

18. A. Gaŭnutdinov, A. Semikhatov, I. Tipunin and B. Fergin - The Kazhdan-Lusztig correspondence for the representation category of the triplet $W$-algebra in logorithmic conformal field theories. (Russian) Teoret. Mat. Fiz. 148 (2006), no. 3, 398-427; translation in Theoret. and Math. Phys. 148 (2006), no. $3,1210-1235$

19. J. Fuchs, C. Schweigert and C. Stigner - Higher genus mapping class group invariants from factorizable Hopf algebras, Adv. Math. 250 (2014), 285-319.

20. N. Geer, J. Kujawa, B. Patureau-Mirand - Generalized trace and modified dimension functions on ribbon categories. Selecta Math. (N.S.) 17 (2011), no. 2, 453-504. 
21. N. Geer, B. Patureau-Mirand - Multivariable link invariants arising from Lie superalgebras of type I. J. Knot Theory Ramifications 19, Issue 1 (2010) 93-115.

22. N. Geer, B. Patureau-Mirand - Polynomial 6j-Symbols and States Sums. Algebraic \& Geometric Topology 11 (2011) 1821-1860.

23. N. Geer, B. Patureau-Mirand - Topological invariants from non-restricted quantum groups. Algebraic \& Geometric Topology, 13 (2013), no. 6, 3305-3363.

24. N. Geer, B. Patureau-Mirand, V. Turaev - Modified quantum dimensions and re-normalized link invariants. Compos. Math. 145 (2009), no. 1, 196-212.

25. N. Geer, B. Patureau-Mirand, V. Turaev - Modified 6j-Symbols and 3-manifold invariants. Adv. Math. 228 (2011), no. 2, 1163-1202.

26. H. Kausch - Extended conformal algebras generated by a multiplet of primary fields. Phys. Lett. B, 259 (1991), no. 4, 448-455.

27. J. Murakami - Colored Alexander Invariants and Cone-Manifolds. Osaka J. Math. Volume 45, Number 2 (2008), 265-564.

28. K. Nagatomo and A. Tsuchiya - The triplet vertex operator algebra $W(p)$ and the restricted quantum group $\bar{U}_{q}\left(s l_{2}\right)$ at $q=e^{\frac{\pi i}{p}}$. Exploring new structures and natural constructions in mathematical physics, Adv. Stud. Pure Math., 61, Math. Soc. Japan, Tokyo, (2011), 1-49.

29. T. Ohtsuki - Quantum invariants. A study of knots, 3-manifolds, and their sets. Series on Knots and Everything, 29. World Scientific Publishing Co., Inc., River Edge, NJ, (2002).

30. R.S. Pierce - Associative algebras. Graduate Texts in Mathematics, 88. Studies in the History of Modern Science, 9. Springer-Verlag, New York-Berlin, (1982).

31. N. Reshetikhin, V.G. Turaev - Ribbon graphs and their invariants derived from quantum groups. Comm. Math. Phys. 127 (1990), no. 1, 1-26.

32. N. Reshetikhin, V.G. Turaev - Invariants of 3-manifolds via link polynomials and quantum groups. Invent. Math. 103 (1991), no. 3, 547-597.

33. V.G. Turaev - Quantum invariants of knots and 3-manifolds. de Gruyter Studies in Mathematics, 18. Walter de Gruyter \& Co., Berlin, (1994).

34. E. Witten - Quantum field theory and Jones polynomial. Comm. Math. Phys. 121 (1989), 351-399.

Institut de Mathématiques Toulouse, 118 route de Narbonne, 31062 Toulouse Cedex 9, France

E-mail address: Francesco.Costantino@math.univ-toulouse.fr

Mathematics \& Statistics, Utah State University, Logan, Utah 84322, USA

E-mail address: nathan.geer@gmail.com

UMR 6205, LMBA, université de Bretagne-Sud, université européenne de Bretagne, BP 573, 56017 VAnnes, France

E-mail address: bertrand.patureau@univ-ubs.fr 